\title{
Explicit de Sitter flux vacua for global string models with chiral matter
}

\author{
Michele Cicoli, ${ }^{a, b, c}$ Denis Klevers, ${ }^{d}$ Sven Krippendorf, ${ }^{e}$ Christoph Mayrhofer, ${ }^{f}$ \\ Fernando Quevedo ${ }^{c, g}$ and Roberto Valandro ${ }^{c, h}$ \\ ${ }^{a}$ Dipartimento di Fisica e Astronomia, Università di Bologna, \\ via Irnerio 46, 40126 Bologna, Italy \\ ${ }^{b}$ INFN, Sezione di Bologna, \\ via Irnerio 46, 40126 Bologna, Italy \\ ${ }^{c} I C T P$, \\ Strada Costiera 11, 34014 Trieste, Italy \\ ${ }^{d}$ Department of Physics and Astronomy, University of Pennsylvania, \\ 209 South 33rd Street, Philadelphia, PA 19104-6396, U.S.A. \\ ${ }^{e}$ Bethe Center for Theoretical Physics and Physikalisches Institut der Universität Bonn, \\ Nussallee 12, 53115 Bonn, Germany \\ ${ }^{f}$ Institut für Theoretische Physik, Universität Heidelberg, \\ Philosophenweg 19, 69120 Heidelberg, Germany \\ ${ }^{g}$ DAMTP, University of Cambridge, \\ Wilberforce Road, Cambridge, CB3 0WA, U.K. \\ ${ }^{h}$ INFN, Sezione di Trieste, \\ Trieste, Italy \\ E-mail: mcicoli@ictp.it, klevers@sas.upenn.edu, \\ krippendorf@th.physik.uni-bonn.de, \\ C.Mayrhofer@thphys.uni-heidelberg.de, F.Quevedo@damtp.cam.ac.uk, \\ rvalandr@ictp.it
}


ABSTRACT: We address the open question of performing an explicit stabilisation of all closed string moduli (including dilaton, complex structure and Kähler moduli) in fluxed type IIB Calabi-Yau compactifications with chiral matter. Using toric geometry we construct Calabi-Yau manifolds with del Pezzo singularities. D-branes located at such singularities can support the Standard Model gauge group and matter content or some close extensions. In order to control complex structure moduli stabilisation we consider CalabiYau manifolds which exhibit a discrete symmetry that reduces the effective number of complex structure moduli. We calculate the corresponding periods in the symplectic basis of invariant three-cycles and find explicit flux vacua for concrete examples. We compute the values of the flux superpotential and the string coupling at these vacua. Starting from these explicit complex structure solutions, we obtain AdS and dS minima where the Kähler moduli are stabilised by a mixture of D-terms, non-perturbative and perturbative $\alpha^{\prime}$ corrections as in the LARGE Volume Scenario. In the considered example the visible sector lives at a $\mathrm{dP}_{6}$ singularity which can be higgsed to the phenomenologically interesting class of models at the $\mathrm{dP}_{3}$ singularity.

KEYwords: Flux compactifications, dS vacua in string theory, Superstring Vacua, Intersecting branes models

ARXIV EPRINT: 1312.0014 


\section{Contents}

1 Introduction 1

2 Effective field theory for complex structure moduli 3

2.1 Kähler potential and flux superpotential 4

$\begin{array}{lll}2.2 & \text { Calculations on explicit CY examples } & 7\end{array}$

$\begin{array}{lll}\text { 2.2.1 Reducing the complex structure moduli by discrete symmetries } & 7\end{array}$

$\begin{array}{lll}2.2 .2 & \text { An explicit example } & 8\end{array}$

3 Explicit model $\quad 11$

3.1 Geometric setup and orientifold involution $\quad 11$

$\begin{array}{lll}3.2 & \text { Visible sector D-branes } & 14\end{array}$

$\begin{array}{ll}3.3 \text { Hidden sector D-branes } & 15\end{array}$

4 Moduli stabilisation $\quad 19$

$\begin{array}{ll}\text { 4.1 Dilaton and complex structure moduli stabilisation } & 19\end{array}$

$\begin{array}{lll}4.2 & \text { Kähler moduli stabilisation } & 23\end{array}$

$\begin{array}{lll}5 & \text { Summary and conclusions } & 29\end{array}$

A Periods and Picard-Fuchs equations $\quad 32$

A.1 Periods as solutions to the Picard-Fuchs equations 32

B More examples $\quad 35$

B.1 Example 2 36

$\begin{array}{ll}\text { B.1.1 The orientifold involution } & 37\end{array}$

$\begin{array}{lll}\text { B.2 Example } 3 & 38\end{array}$

B.2.1 The orientifold involution 40

$\begin{array}{lll}\text { B.3 Example } 4 & 40\end{array}$

$\begin{array}{lll}\text { B.3.1 The orientifold involution } & 42\end{array}$

\section{Introduction}

Constructing realistic string vacua is technically challenging as top-down consistency conditions of string compactifications and bottom-up experimental constraints on the effective field theory lead to strict limitations for any string model. In spite of the difficulty of the task, the bottom-up approach to string model building [1] allows to address various requirements once at a time. Moreover, over the past decade various successful mechanisms, in particular in the context of type IIB string theory, have been established which allow to address moduli stabilisation and realistic D-brane model building [2]. The former can be 
achieved by flux stabilisation of the complex structure moduli and the dilaton (following for example [3]), and a mixture of perturbative and non-perturbative corrections in the effective field theory can be used to stabilise the Kähler moduli $[4,5]$. As far as model building is concerned, D-branes at singularities provide an interesting avenue towards realistic models of particle physics which have been shown to realise extensions of the MSSM with interesting flavour structure (see for instance [6-10]). Similarly local F-theory models provide another interesting class of models with realistic properties [6, 11-14].

For a fully realistic string compactification, all of these successful mechanisms have to be combined in a consistent string set-up. Recently [15-18] we addressed the question of combining Kähler moduli stabilisation with D-brane model building and we continue this program here by also implementing explicitly flux stabilisation of complex structure moduli. Some of the previous accomplishments include:

- Using toric geometry, we constructed explicit compact Calabi-Yau (CY) orientifold compactifications $[19,20]$.

- Chiral matter arises from fractional D3/D7-branes at del Pezzo singularities. These singularities are mapped onto each other by the orientifold involution, realising an invariant setting. The D-brane configuration at these singularities allows for a visible sector including the Standard Model gauge group.

- The geometries contain an additional 'small' (del Pezzo) divisor to generate a nonperturbative superpotential for moduli stabilisation.

- An additional four-cycle class controls the overall size of the compactification which, overall, leads to CY manifolds with $h^{1,1} \geq 4$.

- We performed a full classification of all models of this type with $h^{1,2} \geq 5 \geq h^{1,1} \geq 4$.

- In explicit examples we showed that all known consistency conditions can be satisfied (D-brane tadpole cancellation, Freed-Witten anomalies and K-theory constraints).

- The Kähler moduli can be stabilised as in the LARGE Volume Scenario (LVS), leading to explicit realisations of de Sitter minima by including non-vanishing matter F-term contributions induced by D-term fixing. Supersymmetry is softly broken and can lead to TeV-scale soft masses.

The aim of this paper is to add an additional layer to the above: the explicit flux stabilisation of dilaton and complex structure moduli, while keeping the previously listed properties of the compactification untouched. Typically the accessible CY manifolds in this class of models have a few Kähler moduli but many complex structure moduli, rendering the analysis of the potential of the complex structure moduli very difficult. However, there can be additional symmetries in the complex structure moduli space such that the effective number of complex structure moduli is reduced [22, 23]. In particular we are interested in a subclass of such CY manifolds: those that allow for the Greene-Plesser (GP) construction of the mirror manifold [24]. 
We actively search for such symmetries in the models we previously classified as phenomenologically interesting and identify several examples that allow for this mechanism. In these examples, we explicitly calculate the leading and sub-leading contribution to the prepotential for the special Kähler complex structure moduli space (in the mirror space language, these are polynomial terms and instanton corrections). In one of these examples, we then search for flux minima which set two phenomenologically relevant parameters: the vacuum expectation value (VEV) of the flux superpotential $W_{0}$ and the string coupling $g_{s}$. Due to computational challenges we only have so far a small number of trustable solutions. Nevertheless we find agreement with previous discussions on the approximately uniform distribution of $W_{0}$ and $g_{s}$ in the patch of moduli space with large complex structure moduli [25-27]. We then combine these results with the explicit stabilisation of the Kähler moduli. Hence we work out the first explicit example that allows for closed string moduli stabilisation to de Sitter space and a visible sector on a $\mathrm{dP}_{6}$ singularity. We finally show that the visible sector gauge theory can be related by appropriate VEVs to the interesting class of gauge theories arising from the $\mathrm{dP}_{3}$ singularity.

The rest of this paper is organised as follows. In section 2 we discuss how to explicitly calculate the dilaton and complex structure contribution to the effective supergravity potential (i.e. the prepotential) and present an example for this calculation. For this example, we present a chiral D-brane setup and check for UV consistency conditions in section 3. We stabilise first the dilaton and complex structure moduli, including a short discussion on the statistics of the flux minima, and then the Kähler moduli in section 4 before concluding with section 5. In appendix A we present more details on the analysis of the complex structure moduli space and in appendix B we list further examples that obey our search criteria.

\section{Effective field theory for complex structure moduli}

In this section we explain how one can concretely calculate the Kähler potential and the flux superpotential for the complex structure moduli.

As we review in the following, the computation of both quantities reduces to the problem of finding the periods of a family of CY three-folds $M_{3}$. The periods are completely encoded in the holomorphic prepotential $F$ on the complex structure moduli space of $M_{3}$. The structure and computation of $F$ is addressed most conveniently in the context of mirror symmetry and $N=2$ special geometry, of which we briefly review the basic notions in section 2.1 and refer to appendix A for a more detailed summary. After general remarks, we explicitly compute $F$ and the periods for a concrete CY three-fold $M_{3}$ in section 2.2. This three-fold exhibits the necessary geometrical properties for constructing a phenomenologically promising D-brane setup, cf. section 3 , for which we perform moduli stabilisation explicitly in section 4 .

For an earlier discussion of the $N=2$ special geometry perspective on type II flux compactifications, that parallels parts of the presentation below, along with an analysis of their flux vacua, at generic points and explicitly for certain fluxes at degeneration points of the Calabi-Yau geometries, see [28]. 
We consider Calabi-Yau three-folds $M_{3}$ with a key property, i.e. the presence of a symmetry on its complex structure moduli space, that allows to fix a large number of complex structure moduli without doing any computation, as explained in section 2.2.1. This makes the explicit computation of the periods of $M_{3}$ (performed in section 2.2.2) technically feasible. Explicit computations of periods for other examples of CY threefolds with such symmetries on their complex structure moduli spaces are presented in appendix B.

Before delving into the details of the computations, we begin by introducing some notation and the basic idea behind the stabilisation of dilaton and complex structure moduli which we denote as $\tau=C_{0}+i g_{s}^{-1}$ and $u^{i}, i=1, \ldots, h^{1,2}\left(M_{3}\right)$. The quantised RR- and NSNS-fluxes, $F_{3}=d C_{2}$ and $H_{3}=d B_{2}$, conveniently combined into $G_{3}=F_{3}-\tau H_{3}$, induce the perturbative flux superpotential

$$
W_{\text {flux }}\left(u^{i}, \tau\right)=\int_{M_{3}} \Omega \wedge G_{3} .
$$

It depends on the moduli $\tau, u^{i}$, where the dependence on the $u^{i}$ arises from the holomorphic three-form $\Omega$ on $M_{3}$. The Kähler potential for these moduli reads

$$
K=-\ln \left(i \int \Omega \wedge \bar{\Omega}\right)-\ln (-i(\tau-\bar{\tau}))
$$

Further dependence on $\tau$ and $u^{i}$ appears in the Kähler and superpotential upon the inclusion of open strings and $\alpha^{\prime}$-corrections. From the field theory analysis, these effects turn out to be sub-leading in the process of moduli stabilisation, and so we shall neglect them (see for instance [5]).

Solving the F-term conditions following from (2.1) and (2.2):

$$
D_{u^{i}} W=\left(\partial_{u^{i}}+K_{u^{i}}\right) W=0, \quad D_{\tau} W=\left(\partial_{\tau}+K_{\tau}\right) W=0
$$

generically fixes both $\tau$ and $u^{i}$. We will demonstrate this explicitly in section 4 using the results from section 2.2.2. Notice that even though these are $h^{1,2}+1$ first order equations with the same number of unknowns, there is no a priori guarantee of a solution since they are non-holomorphic. However there are $2 h^{1,2}+2$ flux parameters which generically implies the existence of many solutions which need to be determined numerically. This is of course the main source for the existence of the landscape of type IIB string vacua.

\subsection{Kähler potential and flux superpotential}

In this subsection, we present a brief review of the special geometry of the complex structure moduli space of a CY three-fold and mirror symmetry, following mainly [29].

First, we notice that the complex structure moduli dependence in (2.1) and (2.2) is captured entirely by the holomorphic three-form $\Omega$. In order to compute the dependence of $\Omega$ on the $u^{i}$ explicitly, we introduce the integral symplectic basis $\left(\alpha_{K}, \beta^{K}\right)$ of $H^{3}\left(M_{3}, \mathbb{Z}\right)$ where $K=0, \ldots, h^{2,1}\left(M_{3}\right)$. Then, we can expand the holomorphic three-form $\Omega$ as

$$
\Omega=X^{K} \alpha_{K}-\mathcal{F}_{K} \beta^{K} .
$$


The coefficients are called periods of $\Omega$. They are conveniently combined into the period vector $\Pi^{t}=\left(X^{K}, \mathcal{F}_{K}\right)$. The $X^{K}$ 's are projective coordinates on the complex structure moduli space which has complex dimension $h^{2,1}$. The periods $\mathcal{F}_{K}$ 's are functions of the $X^{K}$ 's. They can be obtained (in a proper symplectic frame) as the partial derivatives of a single holomorphic function $\mathcal{F}$, the projective prepotential, as

$$
\mathcal{F}_{L}=\frac{1}{2} \frac{\partial}{\partial X^{L}}\left(X^{K} \mathcal{F}_{K}\right) \equiv \frac{\partial}{\partial X^{L}} \mathcal{F} .
$$

The prepotential is a homogeneous function of degree two in the $X^{K}$ 's. ${ }^{1}$ We introduce the function

$$
F\left(u^{i}\right)=\left(X^{0}\right)^{-2} \mathcal{F}\left(X^{K}\right),
$$

(that we still call 'prepotential') where we defined the flat coordinates: ${ }^{2}$

$$
u^{i}=\frac{X^{i}}{X^{0}}, \quad i \neq 0
$$

In terms of the prepotential, we can express the period vector as

$$
\Pi=\left(\begin{array}{c}
X^{0} \\
X^{i} \\
\mathcal{F}_{0} \\
\mathcal{F}_{i}
\end{array}\right) \cong\left(\begin{array}{c}
1 \\
u^{i} \\
2 F-u^{i} \partial_{i} F \\
\partial_{i} F
\end{array}\right),
$$

where we used the split $I=(0, i)$ for $i=1, \ldots, h^{1,2}\left(M_{3}\right)$ and in the second equality we normalised $\Omega$ by choosing $X^{0}=1$.

The superpotential and the Kähler potential $K_{\mathrm{cs}}$ for the complex structure moduli (cf. (2.1) and (2.2)) can now be rewritten as

$$
\begin{aligned}
W_{\text {flux }} & =\int G_{3} \wedge \Omega=(f-\tau h)^{t} \cdot \Pi \\
K_{\mathrm{cs}} & =-\ln \left(i \int \Omega \wedge \bar{\Omega}\right)=-\ln \left(i \Pi^{\dagger} \cdot \Sigma \cdot \Pi\right) \\
& =-\ln \left(2 i \operatorname{Im}\left(\bar{X}^{K} \mathcal{F}_{K}\right)\right)=-\ln \left(i\left\|X^{0}\right\|^{2}\left(2 F-2 \bar{F}-(u-\bar{u})^{i}(F+\bar{F})_{i}\right)\right),
\end{aligned}
$$

where "." is the ordinary scalar product and $\Sigma$ is the standard symplectic matrix

$$
\Sigma=\left(\begin{array}{cc}
0 & \mathbf{1} \\
-\mathbf{1} & 0
\end{array}\right) \text {. }
$$

Moreover, the background fluxes can be expanded in the symplectic basis $\left(\alpha_{K}, \beta^{K}\right)$ as

$$
G_{3}=\left(M^{K}-\tau \tilde{M}^{K}\right) \alpha_{K}-\left(N_{K}-\tau \tilde{N}_{K}\right) \beta^{K} .
$$

\footnotetext{
${ }^{1}$ Globally, $\mathcal{F}$ is a section of the square of the vacuum line bundle $\mathcal{L}^{2}$ over the complex structure moduli space.

${ }^{2}$ We deviate here from the usual convention in the mirror symmetry literature, where the flat coordinates are denoted by $t^{i}$, in order to avoid confusions with the Kähler moduli of $M_{3}$.
} 
We have introduced the vectors $f=\Sigma \cdot\left(M^{K}, N_{K}\right)^{t}$ and $h=\Sigma \cdot\left(\tilde{M}^{K}, \tilde{N}_{K}\right)^{t}$ which encode the RR- and NSNS-fluxes. The flux contribution to the D3-tadpole reads

$$
Q_{D 3}=\frac{1}{(2 \pi)^{4}\left(\alpha^{\prime}\right)^{2}} \int_{M_{3}} H_{3} \wedge F_{3}=M^{K} \tilde{N}_{K}-\tilde{M}^{K} N_{K}=-h^{t} \cdot \Sigma \cdot f .
$$

From (2.8) it is now manifest that the key quantity encoding all the information of the periods $\Pi$ is the holomorphic prepotential $F$. Its expansion at large complex structure on $M_{3}$ in terms of the coordinates $u^{i}$ defined in (2.7) is given by (see for example [29]) ${ }^{3}$

$$
F=-\frac{1}{3 !} \mathcal{K}_{i j k} u^{i} u^{j} u^{k}-\frac{1}{2 !} \mathcal{K}_{i j} u^{i} u^{j}+\mathcal{K}_{i} u^{i}+\frac{1}{2} \mathcal{K}_{0}+\sum_{\beta} n_{\beta} \operatorname{Li}_{3}\left(q^{\beta}\right)
$$

It is useful to recall the interpretation of this general form for $F$ from the point of view of the mirror CY three-fold $\tilde{M}_{3}$ and the A-model at large radius/large volume. Here $F$ is the prepotential on the quantum corrected Kähler moduli space of $\tilde{M}_{3}$, with its Kähler moduli related to the flat coordinates $(2.7)$ on $M_{3}$ via the mirror map

$$
u_{\mathrm{B}-\text { model }}^{i} \leftrightarrow t_{\mathrm{A} \text {-model }}^{i}=\int_{\beta^{i}}\left(J+i B_{2}\right)
$$

The two-form $B_{2}$ denotes the NSNS $B$-field, $J$ is the Kähler form on $\tilde{M}_{3}$ and the $\beta^{i}$ are the generators of $H_{2}\left(\tilde{M}_{3}, \mathbb{Z}\right)$. We note that the shift symmetry of $B_{2}$ fixes the $u_{\text {B-model }}^{i}$ uniquely and, in particular, implies their familiar single-logarithmic leading order term. The classical terms in (2.13) are determined by the classical intersections on $\tilde{M}_{3}$ according to [30] (see also [31] for generalisations to higher dimensions):

$$
\begin{aligned}
\mathcal{K}_{i j k} & =\int_{\tilde{M}_{3}} J_{i} \wedge J_{j} \wedge J_{k}, & \mathcal{K}_{i j} & =-\frac{1}{2} \int_{\tilde{M}_{3}} J_{i} \wedge J_{j}^{2} \bmod \mathbb{Z}, \\
\mathcal{K}_{j} & =\frac{1}{2^{2} 3 !} \int_{\tilde{M}_{3}} c_{2}\left(\tilde{M}_{3}\right) \wedge J_{j} \bmod \mathbb{Z}, & \mathcal{K}_{0} & =\frac{\zeta(3)}{(2 \pi i)^{3}} \int_{\tilde{M}_{3}} c_{3}\left(\tilde{M}_{3}\right) .
\end{aligned}
$$

Here $J_{i}$ denote $(1,1)$-forms that are dual to the generators $K_{i}$ of the Kähler cone of $\tilde{M}_{3}$ and $c_{2,3}\left(\tilde{M}_{3}\right)$ denote the second (third) Chern class of the mirror CY. Note that the coefficients $\mathcal{K}_{i j}$ and $\mathcal{K}_{i}$ of the sub-leading terms in the $u^{i}$ are only fixed modulo integers. Fixing them corresponds to a particular choice of integral symplectic basis $\left(\alpha_{K}, \beta^{L}\right)$. ${ }^{4}$ We refer to appendix A for more details on the derivation of (2.15). The last term in (2.13) are the string worldsheet instanton corrections to the A-model. Here we employed the notation $q^{\beta}=e^{2 \pi i d_{j} u^{j}}$ for a class $\beta=d_{i} \beta^{i}$ with degrees $d_{i} \in \mathbb{Z}_{\geq 0}$. The integers $n_{\beta}$ are labeled by the class $\beta$ and are the genus zero Gopakumar-Vafa invariants and the poly-logarithm $\operatorname{Li}_{3}(x)$ is defined as $\operatorname{Li}_{d}(x)=\sum_{n>0} \frac{x^{n}}{n^{d}}$ for an integer $d$.

\footnotetext{
${ }^{3}$ We refer to Equations (A.15) and (A.16) in appendix A for explicit expressions for $\Pi$ and $W_{\text {flux }}$ in terms of the quantities appearing in (2.13).

${ }^{4}$ The freedom in the choice of such a basis is given by the symplectic transformations $\operatorname{Sp}\left(2+2 h^{1,2}, \mathbb{Z}\right)$, w.r.t. which the period vector $\Pi$ transforms in the fundamental representation. For the prepotential (2.13) with the classical terms (2.15) the period vector $\Pi$ is obtained in one particular choice of basis.
} 
On the B-model side of $M_{3}$ the prepotential is classical and, thus, exactly calculable. Both $F$ and the periods, that follow from (2.8), are solutions to the Picard-Fuchs (PF) differential system associated to the family of CY three-folds $M_{3}$ obtained by variation of its complex structure moduli. We refer to appendix A for the construction of the PF system that is straightforward for concrete toric examples. The knowledge of the coefficients in (2.15) is necessary to identify the linear combination of solutions to the PF system that is the prepotential $F$ and for which the expression in (2.8) yields periods on $M_{3}$ in an integral symplectic basis. The instanton corrections of the A-model side are then readily extracted from this solution of the PF system in combination with the mirror map (2.14), which we compute using the Mathematica package Instanton [32].

\subsection{Calculations on explicit CY examples}

In this section we choose a concrete CY three-fold. We explicitly compute the Kähler potential and flux superpotential from the corresponding prepotential $F$. Before delving into the details of these computations, we discuss a general strategy to reduce the typically large complex structure moduli space to a sub-sector of a technically feasible size.

\subsubsection{Reducing the complex structure moduli by discrete symmetries}

As discussed before, a typical phenomenologically interesting CY three-fold features a small number of Kähler moduli and a large number of complex structure moduli. Since it is technically unfeasible to obtain the full period vector for geometries with such a large number of complex structure moduli, we will fix most of the $u$-moduli by a general principle, i.e. without doing a computation. A mechanism to achieve this was suggested and applied in $[22,23,26]$. The basic idea is to consider a CY three-fold ${ }^{5} X_{3}$ which admits subsets of its complex structure moduli space that are invariant under the action of an appropriate discrete symmetry group. ${ }^{6}$ The symmetry group also acts on the periods of $X_{3}$. Switching on fluxes along the invariant three-forms allows to dynamically set the non-invariant complex structure moduli to their fixed point under the group, e.g. zero, by solving the F-term conditions as in (2.3).

In the following, we provide a systematic proof of this argument. ${ }^{7}$ Let us consider a finitely generated symmetry group $G$, as we will do in the rest of this work. Consider a group element $g$ in $G$ that acts non-trivially on a single modulus $u^{s}$, for simplicity, while leaving all other moduli $u^{i}, i \neq s$ invariant. Denoting the group action by $g \cdot, G$-invariant periods and the Kähler potential $K$ obey

$$
\Pi\left(g \cdot u^{s}\right)=e^{f\left(u^{s}\right)} \Pi\left(u^{s}\right), \quad K\left(g \cdot u^{s}\right)=K\left(u^{s}\right)-2 \operatorname{Re}\left(f\left(u^{s}\right)\right),
$$

where we suppressed the dependence on $u^{i}$ for convenience. Here we demand no strict invariance but allow for a non-trivial gauge transformation $e^{f}$, with an appropriate holomorphic function $f\left(u^{s}\right)$. We differentiate both equations in (2.16) with respect to $u^{s}$ to

\footnotetext{
${ }^{5}$ In order to avoid confusion, we denote the CY three-fold and its mirror considered here and in concrete examples by $X_{3}, \tilde{X}_{3}$.

${ }^{6}$ As a necessary condition, this symmetry group has to preserve the CY-condition.

${ }^{7}$ Arguments similar to the following have been carried out independently by A. Klemm.
} 
obtain the following elementary relations:

$$
\begin{aligned}
g\left(\partial_{u^{s}} \Pi\right)(g \cdot x) & =e^{f(x)}\left[\left(\partial_{u^{s}} \Pi\right)(x)+\left(\partial_{u^{s}} f\right)(x) \Pi(x)\right], \\
g\left(\partial_{u^{s}} K\right)(g \cdot x) & =\left(\partial_{u^{s}} K\right)(x)-\left(\partial_{u^{s}} f\right)(x)
\end{aligned}
$$

where $x$ is any value for the variable $u^{s}$. Equipped with these equations it is straightforward to show for the covariant derivative that

$$
g\left(D_{u^{s}} \Pi\right)(g \cdot x)=e^{f(x)}\left(D_{u^{s}} \Pi\right)(x) .
$$

Thus, we infer that $\left(D_{u^{s}} \Pi\right)(\hat{x})=0$ at every fixed point $\hat{x}$ of the action by $g$, i.e. at points with $g \cdot \hat{x}=\hat{x}$, as claimed. This is clear since $g \neq 1$, which is the case as we are assuming that $g$ acts non-trivially on $u^{s}$, and since $e^{f(\hat{x})}=1$, as follows from $(2.16)$ at $u^{s}=\hat{x}{ }^{8}$

In summary, this argument shows that a solution to $D_{u^{s}} W_{\text {flux }}=0$ for a flux superpotential $W_{\text {flux }}$ with fluxes switched on only along invariant three-cycles is given by going to the fixed set under the action of $G$. In other words, for these fluxes the complex structure moduli of $X_{3}$ can be dynamically driven to this $G$-symmetric locus. ${ }^{9}$ Furthermore, the invariant periods, restricted to the $G$-fixed subspace of complex structure moduli, are closed under the PF operators also restricted to this subspace, since they commute with the group $G$. The complex structure moduli space obtained in this way agrees with the one of the CY-quotient $X_{3} / G$.

Concrete examples of such symmetry groups are the Greene-Plesser (GP) orbifold groups $\Gamma$ of the GP construction [24]. This construction provides mirror pairs of CY threefolds formed by the three-fold $X_{3}$ and its quotient $\tilde{X}_{3}=X_{3} / \Gamma$ with all fixed points resolved. The complex structure moduli space of $\tilde{X}_{3}$ is embedded into the complex structure moduli space of $X_{3}$ as the $\Gamma$-invariant subspace and all of the above requirements on $G$ are met by $G \equiv \Gamma$. In particular, the invariant periods restricted to the invariant subspace are simply the periods of the mirror $\tilde{X}_{3}$.

In the following, we will consider a phenomenologically appealing CY three-fold $X_{3}$ with four Kähler moduli and with mirror symmetry realised by the GP construction. We then compute the periods of its quotient CY three-fold $M_{3} \equiv \tilde{X}_{3}=X_{3} / \Gamma$ (cf. the notation of section 2.1) from its PF-system that depends on only four complex structure moduli. We stabilise all non-invariant complex structure moduli of $X_{3}$ at their fixed point under the GP-group $\Gamma$ by the above mechanism. The remaining periods agree with those of the mirror $\tilde{X}_{3}$. Then, stabilisation of complex structure moduli of $X_{3}$ at the $\Gamma$-symmetric point reduces to stabilising explicitly the remaining invariant $u$-moduli.

\subsubsection{An explicit example}

The example we consider is a mirror pair of CY hypersurfaces $\left(X_{3}, \tilde{X}_{3}\right)$ in four-dimensional toric varieties denoted $\mathbb{P}_{\Delta}$ and $\mathbb{P}_{\tilde{\Delta}}$. These toric varieties are specified by their reflexive

\footnotetext{
${ }^{8} e^{f(\hat{x})}=1$ holds whenever the period $\Pi(\hat{x}) \neq 0$. If $\Pi(\hat{x})=0$, then $(2.17)$ still implies $\left(D_{u^{s}} \Pi\right)(\hat{x})=0$ for $g \neq e^{f(\hat{x})}$. In addition, if the invariant subset under $G$ of the complex structure moduli space is not only a point, there always has to be a different invariant period with $\Pi^{\prime}(\hat{x}) \neq 0$ which we can consider.

${ }^{9}$ There might be other solutions to the F-term equations for the $u^{s}$, that we do not consider here.
} 
polytopes that we denote by $\Delta_{4}^{X}$ and $\Delta_{4}^{\tilde{X}}$ for its dual. The vertices of these polytopes are given by the rows of the following matrices:

$$
\Delta_{4}^{X}=\left(\begin{array}{c|rrrr|r}
z_{1} & 1 & 1 & 0 & -1 & D_{1} \\
z_{2} & 1 & 1 & -1 & 0 & D_{2} \\
z_{3} & 1 & 0 & 0 & 0 & D_{3} \\
z_{4} & 1 & 0 & -1 & 1 & D_{4} \\
z_{5} & 0 & 1 & 0 & 0 & D_{5} \\
z_{6} & 0 & 0 & 1 & 0 & D_{6} \\
z_{7} & 0 & 0 & 0 & 1 & D_{7} \\
z_{8} & -1 & -1 & 0 & 0 & D_{8}
\end{array}\right), \quad \Delta_{4}^{\tilde{X}}=\left(\begin{array}{rrrr|r}
-1 & -1 & -1 & -1 & \tilde{D}_{1} \\
2 & -1 & -1 & 2 & \tilde{D}_{2} \\
2 & -1 & 2 & 2 & \tilde{D}_{3} \\
-1 & 2 & -1 & 2 & \tilde{D}_{4} \\
-1 & 2 & 2 & 2 & \tilde{D}_{5} \\
2 & -1 & -1 & -1 & \tilde{D}_{6} \\
2 & -1 & 2 & -1 & \tilde{D}_{7} \\
-1 & 2 & -1 & -1 & \tilde{D}_{8}
\end{array}\right)
$$

Here we have introduced the toric divisors $D_{i}=\left\{z_{i}=0\right\}$ (and $\tilde{D}_{i}$ ) corresponding to the vertices in $\Delta_{4}^{X}$ (and $\Delta_{4}^{\tilde{X}}$ ) in the last column. Note that the polytopes $\Delta_{4}^{X}$ and $\Delta_{4}^{\tilde{X}}$ are congruent. Thus the corresponding toric varieties $\mathbb{P}_{\Delta}$ and $\mathbb{P}_{\tilde{\Delta}}$ differ only by the action of an orbifold group $\Gamma=\mathbb{Z}_{3}^{3}$, and the mirror construction agrees with the GP orbifold construction.

We want to calculate the periods on the complex structure moduli space of the CY three-fold $X_{3}$. Its Hodge data and Euler number read

$$
h^{1,1}\left(X_{3}\right)=4, \quad h^{2,1}\left(X_{3}\right)=70, \quad \chi\left(X_{3}\right)=-132 .
$$

The subspace that is invariant under the GP orbifold group is only four-dimensional and the invariant periods at this $\Gamma$-symmetric point precisely agree with the periods of the mirror $\tilde{X}_{3}$. By application of the argument of section 2.2.1 we reduce the $u$-moduli of $X_{3}$ to this invariant subspace, which agrees with the complex structure moduli space of $\tilde{X}_{3}$. Thus, we present in the remainder of this section the computation of the ten periods on $\tilde{X}_{3}$.

First, we note that there are 16 different star-triangulations of $\Delta_{4}^{X}$ that give rise to eight different $\mathrm{CY}$ phases on $X_{3}$, some of which correspond to multiple phases of the toric variety and others only to one. This can be checked by calculating and comparing the triple intersections on $X_{3}$ for all these 16 toric phases. The triangulations, the intersections and the following calculations were done by means of PALP $[33,34]$ and Sage with the toric geometry package [35]. The Mori cone of a given CY phase is calculated as the intersection of the Mori cones of the corresponding phases of the ambient space, which are related by flops away from the CY hypersurface. For simplicity, we choose a CY phase which arises from only one phase of the ambient toric variety and has a simplicial Mori cone. It is generated by the $\ell^{(i)}$-vectors

$$
\begin{array}{ll}
\ell^{(1)}=(-1,1,1,-1,0,0,0,0), & \ell^{(2)}=(0,-1,0,1,1,0,-1,0), \\
\ell^{(3)}=(0,0,-1,1,0,1,-1,0), & \ell^{(4)}=(1,0,0,0,0,0,1,1) .
\end{array}
$$

The PF-system for $\tilde{X}_{3}$ will be constructed using (A.8) from these $\ell^{(i)}$-vectors. The StanleyReissner ideal in terms of the coordinates $z_{i}$ in (2.19) in this phase reads

$$
\text { SR-ideal : }\left\{z_{2} z_{3}, z_{2} z_{6}, z_{4} z_{5}, z_{4} z_{6}, z_{3} z_{5}, z_{1} z_{7} z_{8}\right\} \text {. }
$$


By duality between curves and divisor:

$$
K_{i} \cdot \ell^{(j)}=\delta_{i}^{j},
$$

the $\ell^{(i)}$-vectors (2.21) also fix the generators $K_{i}$ of the Kähler cone. In the chosen triangulation, the Kähler cone is spanned by the generators

$$
K_{1}=D_{2}+D_{3}+D_{4}, \quad K_{2}=D_{5}, \quad K_{3}=D_{2}+D_{4}, \quad K_{4}=D_{1}+D_{3}+D_{6},
$$

in terms of the toric divisors $D_{i}$ introduced in (2.19).

As a cross-check, we confirm explicitly that there exists a CY phase of the mirror $\tilde{X}_{3}$ that contains this chosen topological phase of $X_{3}$ as a sub-sector of its Kähler moduli space. This confirms, invoking mirror symmetry, that the complex structure moduli space of $\tilde{X}_{3}$ is indeed a sub-sector of the complex structure moduli space of $X_{3}$, as guaranteed by the GP construction.

In the basis (2.24) of the Kähler cone on $X_{3}$ we calculate the triple intersections $\mathcal{K}_{i j k}$. We summarise these intersections on $X_{3}$ in a formal polynomial $\mathcal{C}_{0}=\frac{1}{6 !} \mathcal{K}_{i j k} J_{i} J_{j} J_{k}$ in the dual $(1,1)$-forms $J_{i}$ that reads: ${ }^{10}$

$$
\mathcal{C}_{0}=\frac{3}{2} J_{1}^{2} J_{4}+3 J_{1} J_{2} J_{4}+3 J_{1} J_{3} J_{4}+3 J_{2} J_{3} J_{4}+\frac{9}{2} J_{1} J_{4}^{2}+3 J_{2} J_{4}^{2}+3 J_{3} J_{4}^{2}+\frac{5}{2} J_{4}^{3} .
$$

Analogously we calculate the other classical intersections in (2.15) to be

$$
\begin{aligned}
& \mathcal{K}_{14}=-\frac{9}{2}, \quad \mathcal{K}_{24}=-3, \quad \mathcal{K}_{34}=-3, \quad \mathcal{K}_{44}=-\frac{15}{2}, \quad \mathcal{K}_{41}=-\frac{3}{2}, \\
& \mathcal{K}_{j} J_{j}=\frac{3}{2} J_{1}+J_{2}+J_{3}+\frac{33}{12} J_{4}, \quad \mathcal{K}_{0}=-\frac{\zeta(3)}{(2 \pi i)^{3}} 132,
\end{aligned}
$$

with all other $\mathcal{K}_{i j}=0$. We note that, as before, these relations hold up to integers and the $\mathcal{K}_{i j}$ are symmetric modulo integers.

Finally we obtain the prepotential (2.13) on $\tilde{X}_{3}$ using the intersections (2.25) and (2.26):

$$
\begin{aligned}
F= & -\frac{3}{2}\left(u^{1}\right)^{2} u^{4}-3 u^{1} u^{2} u^{4}-3 u^{1} u^{3} u^{4}-3 u^{2} u^{3} u^{4}-\frac{9}{2} u^{1}\left(u^{4}\right)^{2}-3 u^{2}\left(u^{4}\right)^{2}-3 u^{3}\left(u^{4}\right)^{2} \\
& -\frac{5}{2}\left(u^{4}\right)^{3}+3 u^{1} u^{4}+\frac{3}{2} u^{2} u^{4}+\frac{3}{2} u^{3} u^{4}+\frac{15}{4}\left(u^{4}\right)^{2}+\frac{3}{2} u^{1}+u^{2}+u^{3}+\frac{33}{12} u^{4}-i \zeta(3) \frac{33}{4 \pi^{3}} \\
& +\sum_{\beta} n_{\beta}^{0} \operatorname{Li}_{3}\left(q^{\beta}\right),
\end{aligned}
$$

where $\beta=\left(d_{1}, d_{2}, d_{3}, d_{4}\right)$ in the basis $\beta^{i}$ of effective curves of $H_{2}\left(X_{3}, \mathbf{Z}\right)$ corresponding to the charge vectors $(2.21)$ and $q^{\beta}=e^{2 \pi i d_{i} u^{i}}$. We also introduced the flat coordinates $u^{i}$ on $\tilde{X}_{3}$, that are identified with the Kähler moduli of $X_{3}$ via the mirror map (2.14). The instanton corrections in (2.28) are determined by solving the PF equations on $\tilde{X}_{3}$ that follow from (2.21). First we calculated explicitly the mirror map (2.14) and then identified the prepotential $F$ among the solutions of the PF system by matching the above classical terms (2.25) and (2.26). Then the worldsheet instanton corrections at large volume are

\footnotetext{
${ }^{10}$ We have not chosen the intersection form $I_{3}$ here as $\mathcal{C}_{0}$ is directly related to the cubic terms in $F$.
} 
uniquely determined and read off by comparing to the general large-volume expansion of $F$ in $(2.13){ }^{11}$

Equipped with (2.28) we readily compute the full period vector according to (2.4) and the Kähler potential $K$ as well as the flux superpotential for arbitrary flux choices from (2.9). We refer to appendix A, in particular (A.15) and (A.16), for more explicit formulae of $\Pi$ and $W_{\text {flux }}$ in terms of the prepotential $F$. The explicit stabilisation of dilaton and complex structure moduli on $X_{3}$ at the $\Gamma$-invariant locus is performed in section 4 .

\section{Explicit model}

On the three-fold $X_{3}$ introduced in the previous section, we now construct an explicit chiral D-brane setup which satisfies all stringy consistency requirements, allows for a chiral visible sector and gives rise to a hidden sector suitable for Kähler moduli stabilisation. For this construction of a chiral global model we follow the same philosophy as in $[16,17]$.

In section 3.1 we study the properties of $X_{3}$ and find that it has three $\mathrm{dP}_{6}$ divisors, and two of these divisors can be exchanged by an orientifold involution with $\mathrm{O} 3 / \mathrm{O} 7$ planes. On these exchangeable $\mathrm{dP}_{6}$ divisors, which will be stabilised at zero size, we place a visible sector setup with fractional D3-branes. In section 3.2 we analyse the gauge theory which arises in this sector. For simplicity we concentrate on a setup with no flavour D7-branes. Equipped with this visible sector, we then construct a minimal hidden sector D-brane setup in section 3.3 compatible with all stringy consistency conditions and suitable for moduli stabilisation.

\subsection{Geometric setup and orientifold involution}

The weight matrix for $\Delta_{4}^{X}$ given in $(2.19)$ reads as follows: ${ }^{12}$

\begin{tabular}{|c|c|c|c|c|c|c|c|c|}
\hline$z_{1}$ & $z_{2}$ & $z_{3}$ & $z_{4}$ & $z_{5}$ & $z_{6}$ & $z_{7}$ & $z_{8}$ & $D_{\mathrm{eq}_{Z}}$ \\
\hline 1 & 0 & 0 & 1 & 1 & 1 & 0 & 2 & 6 \\
\hline 1 & 0 & 0 & 0 & 0 & 0 & 1 & 1 & 3 \\
\hline 0 & 1 & 0 & 0 & 0 & 1 & 0 & 1 & 3 \\
\hline 0 & 0 & 1 & 0 & 1 & 0 & 0 & 1 & 3 \\
\hline
\end{tabular}

The Stanley-Reisner ideal for the chosen triangulation of the ambient four-fold is: ${ }^{13}$

$$
\text { SR-ideal : }\left\{z_{2} z_{3}, z_{3} z_{5}, z_{2} z_{6}, z_{2} z_{7}, z_{3} z_{7}, z_{4} z_{5} z_{6}, z_{1} z_{7} z_{8}, z_{1} z_{4} z_{5} z_{8}, z_{1} z_{4} z_{6} z_{8}\right\}
$$

Each column in (3.1) denotes the scaling behaviour of each homogeneous coordinate $z_{i}$ under the four $\mathbb{C}^{*}$-actions of the ambient fourfold. The last column of the table determines

\footnotetext{
${ }^{11}$ The explicit instanton corrections can be found in the accompanying material of this paper on the arXiv.

${ }^{12}$ The triangulation used here is the one relevant for the construction of the CY orientifold of $X_{3}$. It differs from the one in (2.21). The periods on $X_{3}$ are, however, unaffected by this change of triangulation.

${ }^{13}$ Note that out of the 16 triangulations of $\Delta_{4}^{X}$ there are three more which give the same intersection ring on the CY hypersurface.
} 
the CY hypersurface by fixing its defining equation to be of the form

$$
X_{3}: \quad P_{6,3,3,3}\left(z_{i}\right)=0,
$$

with $P_{6,3,3,3}\left(z_{i}\right)$ a polynomial of degrees $(6,3,3,3)$ with respect to the $\left(\mathbb{C}^{*}\right)^{4}$-action of $(3.1)$.

The CY three-fold $X_{3}$ has three $\mathrm{dP}_{6}$ divisors at $z_{2}=0, z_{3}=0$ and $z_{7}=0$. We can take these three divisors as part of an integral 'diagonal' basis of $H^{1,1}\left(X_{3}\right)$ :

$$
\mathcal{D}_{q_{1}}=D_{2}, \quad \mathcal{D}_{q_{2}}=D_{3}, \quad \mathcal{D}_{b}=D_{1}+D_{2}+D_{3}, \quad \mathcal{D}_{s}=D_{7},
$$

i.e. a basis for which the intersection form $I_{3}$ has no cross-terms:

$$
I_{3}=3 \mathcal{D}_{q_{1}}^{3}+3 \mathcal{D}_{q_{2}}^{3}+3 \mathcal{D}_{b}^{3}+3 \mathcal{D}_{s}^{3} .
$$

Expanding the Kähler form $J$ in the basis (3.4) as $^{14}$

$$
J=t_{b} \mathcal{D}_{b}+t_{q_{1}} \mathcal{D}_{q_{1}}+t_{q_{2}} \mathcal{D}_{q_{2}}+t_{s} \mathcal{D}_{s}
$$

the volumes of the four divisors take the form:

$$
\operatorname{Vol}\left(\mathcal{D}_{i}\right) \equiv \tau_{i}=\frac{1}{2} \int_{\mathcal{D}_{i}} J \wedge J=\frac{3}{2} t_{i}^{2},
$$

while the CY volume becomes of strong 'Swiss-cheese' type:

$$
\operatorname{Vol}\left(X_{3}\right) \equiv \mathcal{V}=\frac{1}{6} \int_{X_{3}} J \wedge J \wedge J=\sum_{i} \frac{t_{i}^{3}}{2}=\frac{1}{3} \sqrt{\frac{2}{3}}\left[\tau_{b}^{3 / 2}-\left(\tau_{q_{1}}^{3 / 2}+\tau_{q_{2}}^{3 / 2}+\tau_{s}^{3 / 2}\right)\right]
$$

Note that the minus sign in (3.7) is due to the fact that the Kähler cone conditions for the three $\mathrm{dP}_{6}$ divisors are

$$
t_{q_{1}}<0, \quad t_{q_{2}}<0, \quad t_{s}<0 .
$$

The additional conditions involving $t_{b}$ read as follows:

$$
t_{b}+t_{s}+t_{q_{1}}>0, \quad t_{b}+t_{s}+t_{q_{2}}>0, \quad t_{b}+t_{q_{1}}+t_{q_{2}}>0 .
$$

This Kähler cone is obtained by the union of the four Kähler cones corresponding to the triangulations of $\Delta_{4}^{X}$ which give the same toric intersection numbers on the CY hypersurface. Since this cone is identical to the cone generated by the curves coming from intersections of the hypersurface equation with two toric divisors, we conclude that (3.8) and (3.9) span indeed the Kähler cone of the CY hypersurface [36].

The involution which exchanges the two $\mathrm{dP}_{6}$ divisors $\mathcal{D}_{q_{1}}$ and $\mathcal{D}_{q_{2}}$ is given by:

$$
\sigma: \quad\left(z_{2}, z_{5}\right) \leftrightarrow\left(z_{3}, z_{6}\right)
$$

It is readily checked that it is a symmetry of the weight matrix (3.1). In the following we take the limit in Kähler moduli space in which these two $\mathrm{dP}_{6}$ 's shrink to zero size. The two singularities generated in this way are exchanged under the orientifold involution, as

\footnotetext{
${ }^{14}$ By abuse of notation, we denote the $(1,1)$-forms dual to the divisors (3.4) by the same symbol.
} 
required. The remaining $\mathrm{dP}_{6}$ surfaces at $z_{7}=0\left(\mathcal{D}_{s}\right)$ is kept at finite size. Note that this desired structure of the Kähler moduli arises in the process of moduli stabilisation discussed in section 4 .

The equation defining the CY hypersurface must be symmetric under the orientifold involution. This gives the following restricted equation

$$
P_{6,3,3,3}^{\text {sym }}\left(z_{i}\right) \equiv P_{6,3,3,3}\left(z_{1}, z_{2}, z_{3}, z_{4}, z_{5}, z_{6}, z_{7}, z_{8}\right)+P_{6,3,3,3}\left(z_{1}, z_{3}, z_{2}, z_{4}, z_{6}, z_{5}, z_{7}, z_{8}\right)=0 .
$$

The fixed point set of the involution (3.10) can be determined by considering the (anti-) invariant combinations $y_{i}$ of the homogeneous coordinates $z_{2}, z_{3}, z_{5}$ and $z_{5}$ :

$$
y_{2} \equiv z_{2} z_{3}, \quad y_{3} \equiv z_{5} z_{6}, \quad y_{5} \equiv z_{2} z_{5}+z_{3} z_{6}, \quad y_{6} \equiv z_{2} z_{5}-z_{3} z_{6}
$$

They are all invariant under the orientifold involution, except $y_{6}$ which transforms as $y_{6} \mapsto-y_{6}$. Hence, the hypersurface $y_{6}=0$ belongs to the fixed locus of (3.10). Its intersection with $X_{3}$ gives the following O7-plane:

$$
\text { O7: } z_{2} z_{5}-z_{3} z_{6}=0 \quad \text { with } \quad \mathcal{D}_{O 7}=\mathcal{D}_{b}-\mathcal{D}_{s},
$$

and $\chi\left(\mathcal{D}_{\mathrm{O} 7}\right)=36$. There are also other points in the fixed point set. In fact, we can compensate the minus one factor of $y_{6}$ by using the scaling relations of the toric coordinates (3.1). Taking this into account, we find a codimension-three and a codimension-four fixed locus in the ambient space. The codimension-three locus is

$$
\left\{z_{1}=0, z_{4}=0, y_{5} \equiv z_{2} z_{5}+z_{3} z_{6}=0\right\},
$$

that, once intersected with $X_{3}$, gives three O3-planes. ${ }^{15}$ The codimension-four locus is

$$
\left\{z_{4}=0, \quad y_{5} \equiv z_{2} z_{5}+z_{3} z_{6}=0, \quad z_{7}=0, \quad z_{8}=0\right\} .
$$

This is one point in the ambient space which lies on the symmetric CY hypersurface given by $P_{6,3,3,3}^{\text {sym }}\left(z_{i}\right)=0$ and, therefore, gives one O3-plane. ${ }^{16}$ Thus, in total we have one O7plane and four O3-planes. For the details of the determination of the O3- and O7-planes we refer to $[16,19,20]$, where the fixed-point locus of a holomorphic involution for a different CY three-fold has been determined using identical techniques.

After having derived the fixed point set of our involution, we can calculate, by means of Lefschetz fixed point theorem [37], ${ }^{17}$ the number of complex structure deformations after imposing invariance under (3.10), i.e. $h_{-}^{1,2}$. The fixed point theorem states that the differences between the alternating sum over the odd and the even Betti numbers is just the Euler characteristics of the fixed point set of the involution:

$$
\sum_{i}(-1)^{i}\left(b_{+}^{i}-b_{-}^{i}\right)=\chi\left(O_{\sigma}\right) \quad \text { with } \quad b_{ \pm}^{i}=\sum_{p+q=i} h_{ \pm}^{p, q} .
$$

\footnotetext{
${ }^{15}$ If we intersect (3.14) with the hypersurface equation of $X_{3}$, it reduces to a degree three polynomial which has three solutions each of which is an O3-plane.

${ }^{16}$ The relations (3.15) are four equations in the ambient four-fold that automatically solve the orientifold symmetric equation of the CY three-fold $P_{6,3,3,3}^{\mathrm{sym}}\left(z_{i}\right)=0$.

${ }^{17}$ For applications of this theorem in the physics literature see for instance [38-40].
} 
Our fixed point set $O_{\sigma}$ is given by one O7-plane with $\chi(\mathrm{O} 7)=36$ and four O3-planes with each $\chi(\mathrm{O} 3)=1$ which gives in total 40 for the Euler number of $O_{\sigma}$. In addition, we know that $h_{-}^{1,1}=1, h_{+}^{1,1}=3, h^{2,1}=70, h^{3,0}=h_{-}^{3,0}=1\left(\Omega \in H_{-}^{3,0}\left(X_{3}\right)\right)$ and that we are dealing with a proper CY three-fold, i.e. $h^{1,0}=h^{2,0}=0$. These data together with (3.16) give us $h_{-}^{2,1}=43$ and $h_{+}^{2,1}=27$.

Next, we consider in more detail the GP orbifold group $\Gamma$ used for the construction of the mirror $\tilde{X}_{3}$. This is essential in order to understand the complex structure moduli space of $X_{3}$ that is both invariant under $\Gamma$ and the orientifold involution (3.10). The GP group $\Gamma=\mathbb{Z}_{3}^{3}$ has the following generators:

$$
\begin{aligned}
\gamma_{1}: & \left(z_{1}, z_{2}, z_{3}, z_{4}, z_{5}, z_{6}, z_{7}, z_{8}\right) \mapsto\left(z_{1}, z_{2}, z_{3}, z_{4}, z_{5}, z_{6}, e^{2 \pi i / 3} z_{7}, e^{4 \pi i / 3} z_{8}\right) \\
\gamma_{2}: & \left(z_{1}, z_{2}, z_{3}, z_{4}, z_{5}, z_{6}, z_{7}, z_{8}\right) \mapsto\left(z_{1}, z_{2}, z_{3}, z_{4}, e^{2 \pi i / 3} z_{5}, z_{6}, e z_{7}, e^{4 \pi i / 3} z_{8}\right) \\
\gamma_{3}: & \left(z_{1}, z_{2}, z_{3}, z_{4}, z_{5}, z_{6}, z_{7}, z_{8}\right) \mapsto\left(z_{1}, z_{2}, z_{3}, z_{4}, z_{5}, e^{4 \pi i / 3} z_{6}, e^{2 \pi i / 3} z_{7}, z_{8}\right)
\end{aligned}
$$

From this, we infer that the orientifold invariant CY equation $P_{6,3,3,3}^{\text {sym }}=0$ in (3.11) takes the following restricted form, if (3.17) is imposed to be a symmetry as well:

$$
\begin{aligned}
P_{6,3,3,3}^{\mathrm{rd}}\left(z_{i}\right)= & z_{1}^{3} z_{2}^{3} z_{5}^{3}+z_{1}^{3} z_{3}^{3} z_{6}^{3}+z_{2}^{3} z_{4}^{3} z_{5}^{3} z_{7}^{3}+z_{3}^{3} z_{4}^{3} z_{6}^{3} z_{7}^{3}+z_{5}^{3} z_{6}^{3} z_{7}^{3}+z_{8}^{3} \\
& +\rho_{1} z_{2}^{3} z_{3}^{3} z_{4}^{6} z_{7}^{3}+\rho_{2} z_{1}^{3} z_{2}^{3} z_{3}^{3} z_{4}^{3}+\rho_{3} z_{1} z_{2} z_{3} z_{4} z_{5} z_{6} z_{7} z_{8} .
\end{aligned}
$$

In this equation, we have fixed completely the reparametrisation invariance. Hence, the remaining undetermined three coefficients are related to the complex structure moduli that survive both the orientifold and the discrete symmetry projection. Note that before imposing the orientifold projection we have four of them, as expected from section 2.2.2.

\subsection{Visible sector D-branes}

Let us discuss the field theory on the D3-branes at the $\mathrm{dP}_{6}$ singularity of $X_{3} / \sigma$. We construct the visible sector of our model by placing $N_{D 3}$ D3-branes on top of the $\mathrm{dP}_{6}$ singularity at $z_{2}=0$ (plus their $N_{D 3}$ images at the singularity at $z_{3}=0$ ) of $X_{3}$. As explained above, these singularities are generated by shrinking the two $\mathrm{dP}_{6}$ divisors, $\mathcal{D}_{q_{1}}$ and $\mathcal{D}_{q_{1}}$, exchanged by the orientifold involution. We do not consider additional flavour D7-branes for simplicity, because their presence would also affect the hidden D7-brane setup described in the next section. ${ }^{18}$

Let us briefly comment on how this $\mathrm{dP}_{6}$ singularity can be the basis for model building. As pointed out in [42] there exists a gauge theory description of the $\mathrm{dP}_{6}$ singularity which locally is a $\mathbb{C}^{3} / \mathbb{Z}_{3} \times \mathbb{Z}_{3}$ orbifold singularity. We use this gauge theory description in terms of the $\mathbb{C}^{3} / \mathbb{Z}_{3} \times \mathbb{Z}_{3}$ orbifold as the starting point for our model building discussion. Using dimer techniques, we find the dimer and quiver diagram as shown in figure 1. Nodes in the quiver correspond to $\mathrm{U}\left(N_{i}\right)$ gauge theories, arrows to bi-fundamental fields. The dimer can be

\footnotetext{
${ }^{18}$ If the local gauge theory construction involves flavour D7-branes, one has also to add some D7-branes which can play their rôle in the global configuration. In this case, the D7-tadpole induced by the O7-plane should not be completed saturated by the stack of D7-branes on top of the O7 (see [17, 18, 41]). In the presence of flavour D7-branes, we might keep $N<4$ D7-branes on top of the O7-plane and the form of the FI-term in (3.31) would still be the same, only the rank of the $\mathrm{U}(N)$ gauge group would differ.
} 

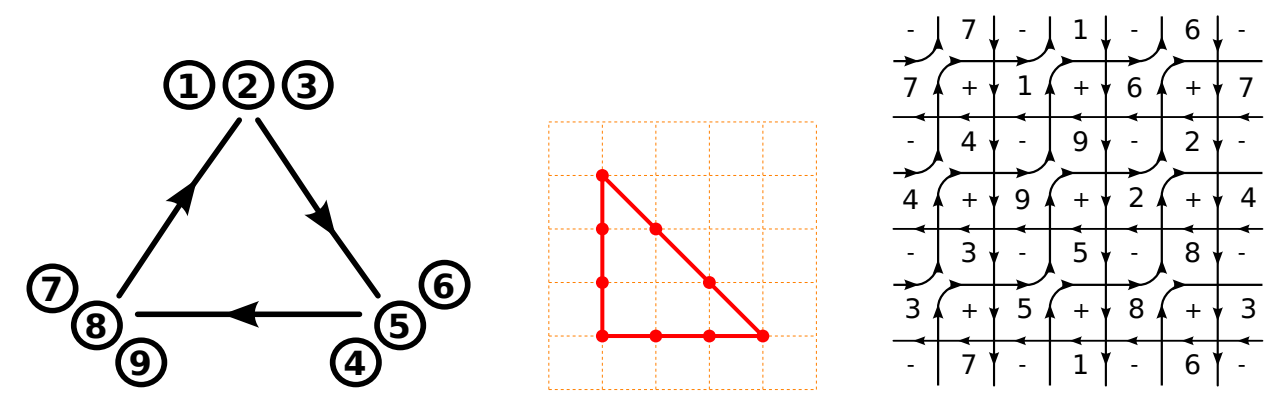

Figure 1. From left to right, the quiver diagram, the toric diagram and the dimer diagram for the $\mathbb{C}^{3} / \mathbb{Z}_{3} \times \mathbb{Z}_{3}$ orbifold singularity.

obtained from the toric diagram using various inverse algorithms (e.g. [43-45]). Numbered faces in the dimer correspond to gauge groups, common nodes of faces to bi-fundamental matter, and the " \pm " faces correspond to superpotential terms. From the dimer in figure 1 we can then read off the following superpotential

$$
\begin{aligned}
W= & X_{14} Y_{47} Z_{71}-X_{14} Y_{49} Z_{91}-X_{15} Y_{57} Z_{71}+X_{15} Y_{58} Z_{81}-X_{16} Y_{68} Z_{81} \\
& +X_{16} Y_{69} Z_{91}-X_{24} Y_{47} Z_{72}+X_{24} Y_{48} Z_{82}-X_{25} Y_{58} Z_{82}+X_{25} Y_{59} Z_{92} \\
& +X_{26} Y_{67} Z_{72}-X_{26} Y_{69} Z_{92}-X_{34} Y_{48} Z_{83}+X_{34} Y_{49} Z_{93}+X_{35} Y_{57} Z_{73} \\
& -X_{35} Y_{59} Z_{93}-X_{36} Y_{67} Z_{73}+X_{36} Y_{68} Z_{83} .
\end{aligned}
$$

The fields $X_{i j}, Y_{k l}, Z_{m n}$ are labelled by different letters for each of the three different arrows in the quiver in figure 1 and the indices in their labelling denote the transformation behaviour as bi-fundamental fields $\left(N_{i}, \bar{N}_{j}\right)$. This gauge theory can be related to phenomenologically interesting gauge theories via higgsing. In particular by assigning a VEV to $X_{14}, Y_{58}$, and $Z_{73}$, we find the following superpotential after integrating out all states with superpotential mass terms:

$$
\begin{aligned}
W= & -X_{16} X_{21} Y_{59} Y_{65} Z_{32} Z_{93}+X_{16} X_{31} Y_{69} Z_{93}+X_{21} Y_{15} Y_{59} Z_{92} \\
& +X_{26} Y_{65} Z_{32} Z_{53}-X_{26} Y_{69} Z_{92}-X_{31} Y_{15} Z_{53}
\end{aligned}
$$

This process is also visualised in figure 2 which shows the dimer diagram after assigning these VEVs and the associated quiver diagram.

This gauge theory is the gauge theory of $\mathrm{dP}_{3}$ which has proven to be phenomenologically very appealing [10]. Models based on the Pati-Salam gauge group have been constructed in [10] where it was shown that they can, in the presence of flavour D7-branes, give rise to a breakdown to the SM gauge group, an appealing flavour structure for quarks and leptons, the absence (respectively sufficient suppression) of proton decay and a viable $\mu$ term.

\subsection{Hidden sector D-branes}

In this section we discuss the $\mathrm{D}$-brane setup away from the $\mathrm{dP}_{6}$-singularity. This includes a discussion of both the D7-branes necessary for D7 tadpole cancellation as well as possible non-perturbative effects from D3-brane instantons and gaugino condensates. 

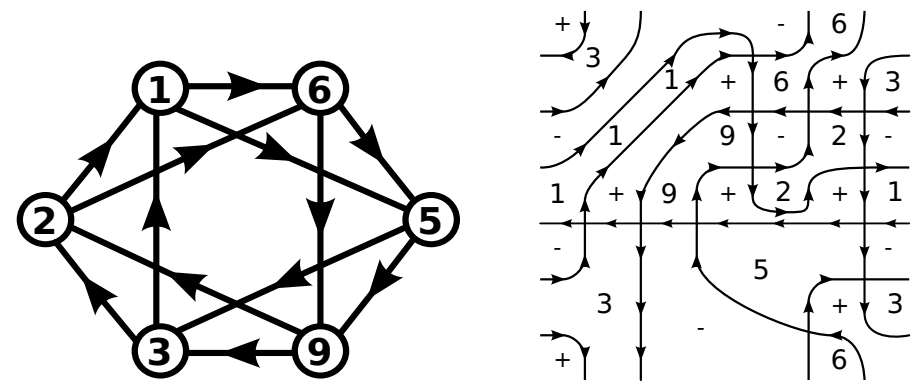

Figure 2. After assigning VEVs for the fields $X_{14}, Y_{58}, Z_{73}$ the matter content shown in the quiver diagram on the left side remains. The resulting dimer after the higgsing is shown on the right side. The remaining gauge theory is that of $\mathrm{dP}_{3}$.

E3 instantons. We need to consider D-branes which generate non-vanishing terms in the non-perturbative superpotential. This is relevant in order to fix some of the Kähler moduli as we will see in section 4.2. The non-perturbative superpotential is generated by E3-instantons and/or gaugino condensation on stacks of D7-branes wrapping four-cycles in the CY three-fold (see for instance [46] for a review). In the LVS the leading contribution comes from a shrinkable rigid divisor (e.g. del Pezzo), if this can support non-perturbative effects.

In our model based on the CY orientifold $X_{3} / \sigma$, we do not have D7-branes wrapping the (orientifold invariant) del Pezzo divisor $\mathcal{D}_{s}$, because the O7-plane charge on the divisor (3.13) cannot compensate the D7 tadpole of these D7-branes. Hence the possible leading non-perturbative effect arises from $O(1)$ E3-instantons wrapping the divisor $\mathcal{D}_{s}$ which is mapped to itself under the orientifold involution $\sigma$. Since the fermionic zero modes of such instantons are projected out, whether the E3's contribute to the superpotential depends only on their world-volume fluxes. The divisor $\mathcal{D}_{s}$ is non-spin and hence Freed-Witten (FW) anomaly cancellation enforces a non-zero gauge flux [47]:

$$
F_{E 3}+\frac{c_{1}\left(\mathcal{D}_{s}\right)}{2} \in H^{2}\left(\mathcal{D}_{s}, \mathbb{Z}\right)
$$

This quantisation implies that the gauge invariant field strength $\mathcal{F}_{E 3}=F_{E 3}-i_{\mathcal{D}_{s}}^{*} B$ is non-zero, if the pullback $i_{\mathcal{D}_{s}}^{*} B$ of the NSNS $B$-field to the world-volume $\mathcal{D}_{s}$ of the brane is zero. Hence, an $O(1)$ E3-instanton wrapping such a divisor is not invariant under the orientifold involution and is projected out. ${ }^{19}$

If we insisted on having $\iota_{\mathcal{D}_{s}}^{*} B=0$, the leading contribution to the superpotential arises from a rank-two E3-instanton [48], which we will however not consider in the following. In fact, in the LVS a leading non-perturbative contribution to the superpotential from rankone E3-instantons is phenomenologically preferred compared to rank-two E3-instantons. The volume in this minimum is fixed to be $\mathcal{V} \sim\left|W_{0}\right| e^{a \tau_{s}}$. For a rank-one E3-instanton we have $a=2 \pi$, while for a rank-two E3-instanton $a=4 \pi$. Since $\tau_{s}>1$ and $\left|W_{0}\right| \sim \mathcal{O}(1)$,

\footnotetext{
${ }^{19} \mathrm{~A}$ two-form $F_{E 3}$ satisfying (3.21) is always even when $\mathcal{D}_{s}$ has zero intersection numbers with the odd $h_{-}^{1,1}$ divisors, as it happens in the considered example.
} 
the second option would fix the volume of the $\mathrm{CY}$ at a too large value, rendering the phenomenologically relevant scale too small. We will comment on this in section 4.2.

Thus we choose a non-zero B-field

$$
i_{\mathcal{D}_{s}}^{*} B=\frac{\mathcal{D}_{s}}{2},
$$

so that we can pick a gauge flux $F_{E 3}$ such that $\mathcal{F}_{E 3}=0$ [49]. In this case the $O(1)$ E3-instanton is invariant and survives the orientifold projection.

All other possible E3-instantons wrap divisors that, in the LVS minimum, have a larger size compared to $\mathcal{D}_{s}$. Thus, their contributions to the superpotential are exponentially suppressed and can be consistently neglected.

Hidden D7-branes. To cancel the D7-tadpole introduced by the O7-plane, we place four D7-branes (plus their images) on top of the O7-plane locus $\mathcal{D}_{O 7}$ given in (3.13). This generates a hidden $\mathrm{SO}(8)$ gauge group. The number of deformation moduli of such D7branes is $h^{0,2}\left(\mathcal{D}_{O 7}\right)=2$. There are no Wilson-line moduli as $h^{0,1}\left(\mathcal{D}_{O 7}\right)=0$.

If the $B$-field is zero, this group is broken to $\mathrm{U}(4)$ by a properly quantised non-zero gauge flux. In fact, FW anomaly cancellation implies the flux to be quantised such that

$$
F_{D 7}+\frac{c_{1}\left(\mathcal{D}_{D 7}\right)}{2} \in H^{2}\left(\mathcal{D}_{D 7}, \mathbb{Z}\right)
$$

Since $\mathcal{D}_{D 7}=\mathcal{D}_{O 7}$ is an odd cycle, the flux is necessary non-zero. ${ }^{20}$

We choose in the following the minimal choice for the $B$-field that satisfies (3.22):

$$
B=-\frac{c_{1}\left(\mathcal{D}_{s}\right)}{2}=\frac{\mathcal{D}_{s}}{2} .
$$

We consider a gauge flux $F_{D 7}$ on the brane world-volume which is a linear combination of pulled back two-forms from the CY three-fold. Since the intersections of $X_{3}$, cf. (3.2), imply that $i_{\mathcal{D}_{O 7}}^{*} \mathcal{D}_{q_{1}}=i_{\mathcal{D}_{O 7}}^{*} \mathcal{D}_{q_{2}}=0$, the flux can only be a linear combination of $i_{\mathcal{D}_{O 7}}^{*} \mathcal{D}_{b}$ and $i_{\mathcal{D}_{O 7}}^{*} \mathcal{D}_{s}$ which we still call $\mathcal{D}_{b}$ and $\mathcal{D}_{s}$ by abuse of notation. By taking into account $(3.23),(3.25)$ and $c_{1}\left(\mathcal{D}_{O 7}\right)=-\mathcal{D}_{b}+\mathcal{D}_{s}$, the generic invariant and quantised flux on the brane is:

$$
\mathcal{F}_{D 7}=F_{D 7}-i_{\mathcal{D}_{O 7}}^{*} B=\left(n_{b}+\frac{1}{2}\right) \mathcal{D}_{b}+\left(n_{s}-1\right) \mathcal{D}_{s}
$$

with $n_{b}, n_{s} \in \mathbb{Z}$. On the image stack the flux is the opposite. Given that the flux along $\mathcal{D}_{b}$ cannot be cancelled due to our $B$-field choice, $\mathcal{F}_{D 7}$ is different from zero. This has a few consequences:

${ }^{20}$ Alternatively, if we choose the $B$-field to be (consistently with (3.22)):

$$
B=-\frac{c_{1}\left(\mathcal{D}_{O 7}\right)}{2}=\frac{\mathcal{D}_{b}}{2}-\frac{\mathcal{D}_{s}}{2},
$$

the gauge invariant flux can be set to zero, $\mathcal{F}_{D 7}=F_{D 7}-i_{\mathcal{D}_{O 7}}^{*} B=0$. Thus, the $\mathrm{SO}(8)$ gauge group is unbroken. By switching on a proper rigidifying flux, we could lift the deformation moduli $[26,50,51]$. Hence, for the $B$-field (3.24), we have a pure super Yang-Mills theory in the hidden sector which undergoes gaugino condensation. However, as we shall see in section 4.2, this setup would not give rise to any D-term associated to an anomalous $\mathrm{U}(1)$ living on $\mathcal{D}_{D 7}$ which is a necessary ingredient to obtain de Sitter vacua. 
- $\mathcal{F}_{D 7} \neq 0$ breaks $\mathrm{SO}(8) \rightarrow \mathrm{SU}(4) \times \mathrm{U}(1)$ where the anomalous $\mathrm{U}(1)$ factor gives rise to a D-term contribution to the scalar potential. The corresponding Fayet-Iliopoulos (FI) term reads [52]:

$$
\xi_{D 7}=\frac{1}{\mathcal{V}} \int_{\mathcal{D}_{D 7}} J \wedge \mathcal{F}_{D 7}=\frac{3}{\mathcal{V}}\left[\left(2 n_{b}+1\right) \frac{t_{b}}{2}+\left(1-n_{s}\right) t_{s}\right]
$$

- $\mathcal{F}_{D 7} \neq 0$ generates massless chiral matter living on the hidden sector D7-branes. The number of chiral fields in the antisymmetric representation of $U(4)$ is (see for instance [53]):

$$
I_{\mathrm{U}(4)}=\frac{1}{2} I_{D 7-D 7^{\prime}}+I_{D 7-O 7}
$$

where:

$I_{D 7-D 7^{\prime}}=\int_{\mathcal{D}_{D 7} \cap \mathcal{D}_{D 7^{\prime}}}\left(\mathcal{F}_{D 7}-\mathcal{F}_{D 7^{\prime}}\right)=2 \int_{X_{3}} \mathcal{D}_{D 7} \wedge \mathcal{D}_{D 7} \wedge \mathcal{F}_{D 7}=3\left(2 n_{b}+1\right)-6\left(1-n_{s}\right)$, since $\mathcal{D}_{D 7^{\prime}}=\mathcal{D}_{D 7}$ and $\mathcal{F}_{D 7^{\prime}}=-\mathcal{F}_{D 7}$, while:

$$
I_{D 7-O 7}=\int_{\mathcal{D}_{D 7} \cap \mathcal{D}_{O 7}} \mathcal{F}_{D 7}=\frac{1}{2} I_{D 7-D 7^{\prime}}
$$

as $\mathcal{D}_{O 7}=\mathcal{D}_{D 7}$. Hence (3.28) reduces to $I_{\mathrm{U}(4)}=I_{D 7-D 7^{\prime}}$.

- $\mathcal{F}_{D 7} \neq 0$ induces chiral states between the D7-stack and the E3-instanton. The number of E3 zero-modes in the fundamental representation of the hidden U(4) gauge group is $[46,54-56]$ :

$$
I_{D 7-E 3}=\int_{\mathcal{D}_{D 7} \cap \mathcal{D}_{s}}\left(F_{D 7}-F_{\mathrm{E} 3}\right)=\int_{\mathcal{D}_{D 7} \cap \mathcal{D}_{s}} \mathcal{F}_{D 7}=3\left(1-n_{s}\right) .
$$

The presence of chiral E3 zero-modes can prevent the contribution of the E3 instanton to the superpotential. When such chiral states are present, we only obtain a non-zero instanton contribution to the superpotential when the scalar component of the chiral superfield prefactor obtains a non-zero VEV $[57,58]$. Therefore, to avoid this possible problem, we consider $n_{s}=1$ so that $I_{D 7-E 3}$ vanishes. We finally have:

$$
I_{D 7-E 3}=0, \quad I_{\mathrm{U}(4)}=3\left(2 n_{b}+1\right) \quad \text { and } \quad \xi_{D 7}=\frac{I_{\mathrm{U}(4)}}{2} \frac{t_{b}}{\mathcal{V}}
$$

The D3-charge of this configuration (including the four O3-planes) is:

$$
Q_{D 3}^{\mathrm{hid}}=-\frac{n_{O 3}}{4}-\frac{1}{2} \chi\left(\mathcal{D}_{O 7}\right)+8\left(-\frac{1}{2} \int_{\mathcal{D}_{O 7}} \mathcal{F}_{D 7}^{2}\right)=-19-3\left(1+2 n_{b}\right)^{2}=-22
$$

where at the end we set $n_{b}=-1$. 


\section{Moduli stabilisation}

We have now collected all the ingredients to proceed with the discussion of moduli stabilisation in the explicit example. In section 4.1 we stabilise of the dilaton and complex structure moduli using background fluxes. In section 4.2 we come then to the stabilisation of the Kähler moduli. Our discussion is based on the geometry of the CY three-fold $X_{3}$ introduced in section 2.2.2 and the associated brane setup discussed in the previous section. The results are compared to the ones obtained for the three-fold $\mathbb{P}_{[1,1,1,6,9]}^{4}[18]$. Equipped with the explicit flux solutions, we then proceed with the stabilisation of the Kähler moduli and find explicit de Sitter minima with TeV-scale soft masses.

\subsection{Dilaton and complex structure moduli stabilisation}

In section 2.2.2 we obtained the pre-potential (2.28) and all the corresponding periods. Let us now proceed with the stabilisation of the dilaton and complex structure moduli. We turn on flux only along three-cycles which are invariant under $\mathbb{Z}_{3}^{3}$. The complex structure moduli which are not invariant under the discrete symmetry are stabilised such that the Calabi-Yau is symmetric under the GP-group $\mathbb{Z}_{3}^{3}$, i.e. zero, following exactly the arguments outlined in section 2.2.1. Hence, they are not present in our prepotential.

We are then left with the stabilisation of the (four) invariant $u$-moduli and the dilaton. The imaginary self-dual (ISD) condition for the fluxes halves the number of independent flux choices. We consider in the following fluxes with a positive D3 tadpole, which is a necessary condition for the ISD condition. We specify fluxes of the form:

$$
\left(\begin{array}{c}
\tilde{M}_{K} \\
\tilde{N}^{K}
\end{array}\right)=\left(\begin{array}{c}
-N^{K} \\
M_{K}
\end{array}\right)
$$

which leaves us with $2 \times 4+2=10$ independent flux parameters. Indeed, in this parametrisation the D3 tadpole (2.12) becomes automatically positive semi-definite, $Q_{D 3}=$ $\sum_{K}\left(\left(\tilde{M}_{K}\right)^{2}+\left(\tilde{N}^{K}\right)^{2}\right)$. Furthermore, some flux configurations are related by $\operatorname{SL}(2, \mathbb{Z})$ transformations which leads to further redundancies:

$$
\left(\begin{array}{c}
\tilde{M}_{K} \\
\tilde{N}^{K}
\end{array}\right) \simeq\left(\begin{array}{c}
-\tilde{M}_{K} \\
-\tilde{N}^{K}
\end{array}\right) \simeq\left(\begin{array}{c}
-\tilde{N}^{K} \\
\tilde{M}_{K}
\end{array}\right) \simeq\left(\begin{array}{c}
\tilde{N}^{K} \\
-\tilde{M}_{K}
\end{array}\right) .
$$

We are interested in $\mathrm{SL}(2, \mathbb{Z})$ invariant flux configurations with a tadpole $Q_{D 3} \leq 22-2 N_{D 3}$, since the D3-tadpole of our setup in section 3.3 was $-\left(22-2 N_{D 3}\right)$ (cf. (3.32) after including the contribution from the D3-branes at the singularities).

Looking at the prepotential (2.28) we observe an additional symmetry between the complex structure moduli $u_{2}$ and $u_{3}$. This is related to the orientifold involution. ${ }^{21}$ To consider orientifold invariant configurations, we need to restrict our analysis to minima with $u_{2}=u_{3}$ and flux choices which have equal fluxes corresponding to these two moduli.

\footnotetext{
${ }^{21}$ It is worthwhile to note that the generators $K_{2}$ and $K_{3}$, cf. (2.24), correspond to the monomials $\alpha_{8} z_{1}^{3} z_{2}^{3} z_{5}^{3}$ and $\alpha_{7} z_{1}^{3} z_{3}^{3} z_{6}^{3}$ in the hypersurface equation (3.18). Therefore, setting $u_{2}=u_{3}$ is linked with the involution (3.10) of the CY three-fold.
} 
This reduces the problem to eight independent flux choices and three complex structure moduli.

From the prepotential (2.28) and a given flux choice we can calculate the scalar potential and, in particular, the covariant derivatives $D_{i} W$. We are interested in the solutions of $D_{i} W=0$ and would like to find such minima numerically as an analytic treatment seems infeasible. To minimise the potential numerically in an efficient and reliable way, we limit ourselves to the prepotential without the instanton corrections at first (in the mirror symmetric language). Given a minimum for this setup, we check in a second step whether the instanton corrections are small enough to keep this minimum stable at the computed values of $u_{i}$.

To solve this problem numerically, we use the openly available package Paramotopy [59], which allows to scan over various flux choices more efficiently. Paramotopy relies on Bertini [60] which is a homotopy continuation solver that produces solutions to polynomial systems. This numerical analysis only identifies isolated minima but neglects continuous ones. As we have to treat the conjugate of a complex structure modulus as an independent variable during the numerical search with this code, we stress that this might neglect various minima which are not found because they might be realised as continuous minima in this larger system under consideration.

To simplify the system (2.3) further we can solve the dilaton equation explicitly. Indeed, we find that

$$
\tau=\frac{f \cdot \bar{\Pi}}{h \cdot \bar{\Pi}}
$$

and a similar expression for its complex conjugate $\bar{\tau}$ solve the F-term condition

$$
0=\partial_{\tau} W+W \partial_{\tau} K=-h \cdot \Pi+(f-\tau h) \cdot \Pi\left(-\frac{1}{\tau-\bar{\tau}}\right)
$$

where we used the Kähler potential (2.2) and the notation introduced in (2.9).

We can use this result to simplify the covariant derivatives for the complex structure moduli as follows. First we expand the first equation in (2.3) explicitly as

$$
0=\partial_{i} W+W \partial_{i} K=(f-\tau h) \cdot \partial_{i} \Pi-(f-\tau h) \cdot \Pi \frac{\Pi^{\dagger} \cdot \Sigma \cdot \partial_{i} \Pi}{\Pi^{\dagger} \cdot \Sigma \cdot \Pi} .
$$

Next, we cancel the denominator, then use (4.3) and cancel denominators again to obtain

$$
0=\left(\Pi^{\dagger} \cdot \Sigma \cdot \Pi\right)\left(f \cdot \partial_{i} \Pi h \cdot \bar{\Pi}-f \cdot \bar{\Pi} h \cdot \partial_{i} \Pi\right)-(f \cdot \Pi h \cdot \bar{\Pi}-f \cdot \bar{\Pi} h \cdot \Pi)\left(\Pi^{\dagger} \cdot \Sigma \cdot \partial_{i} \Pi\right) .
$$

We use this equation as an input for the numerical minimisation which is a set of polynomial equations. In complete analogy we can obtain the input for the covariant derivatives with respect to the complex conjugated variables.

Under the above assumptions, we have searched for minima for each D3 tadpole in the range between $Q_{D 3}=10, \ldots, 20$ for 100 randomly chosen flux configurations. On average we find 2.59 solutions for a single flux configuration. At first many solutions correspond to solutions outside the fundamental domain and with large instanton corrections, but we can use the $\operatorname{SL}(2, \mathbb{Z})$ freedom to transform solutions into the fundamental domain. We show in 


\begin{tabular}{|c|c|c|c|c|c|c|c|}
\hline$Q_{D 3}$ & $\left(\tilde{N}_{i}, \tilde{M}_{i}\right)$ & $u_{1}$ & $u_{2,3}$ & $u_{4}$ & $\tau$ & $g_{s}$ & $\left|W_{0}\right|$ \\
\hline 19 & $(1,-3,0,0,0,0,2,-1)$ & $4.39-1.22 i$ & $19.3+0.971 i$ & $-21.6+1.18 i$ & $2.21+2.58 i$ & 0.39 & 954.4 \\
\hline 14 & $(1,1,1,1,3,0,0,0)$ & $-5.99+2.69 i$ & $4.09+1.59 i$ & $-0.115-0.0581 i$ & $-2.76+1.24 i$ & 0.8 & 82.66 \\
\hline 14 & $(1,0,0,1,1,-3,1,0)$ & $4.72+2.7 i$ & $-3.92+1.94 i$ & $0.176-0.0468 i$ & $4.14+1.32 i$ & 0.75 & 54.03 \\
\hline 15 & $(2,0,2,1,0,0,-1,0)$ & $28 .+3.3 i$ & $-11.4+2.62 i$ & $0.331-0.0291 i$ & $6.72+1.3 i$ & 0.77 & 55.85 \\
\hline 15 & $(1,2,1,1,1,2,-1,0)$ & $1.49+0.861 i$ & $-1.22+1.77 i$ & $-0.201-0.0276 i$ & $-2.41+2.22 i$ & 0.45 & 36.44 \\
\hline 18 & $(1,2,0,2,2,2,0,-1)$ & $1.13+0.473 i$ & $-0.327+2.02 i$ & $-0.583+0.103 i$ & $-1.5+3.44 i$ & 0.29 & 126.5 \\
\hline
\end{tabular}

Table 1. Summary of all solutions which satisfy $0<g_{s}<1$ and obey $\left|F_{\text {inst }}\right| /|F|<1$, $\max _{i}\left(\left|F_{\text {inst }}^{i}\right|\right) /|F|<1$. The flux configuration is given where the third and respectively seventh entry denote the flux quanta $\tilde{N}_{2}=\tilde{N}_{3}$ respectively $\tilde{M}_{2}=\tilde{X}_{3}$ which are chosen to be the same as described in the main text.

\begin{tabular}{|c|c|c|c|c|c|c|}
\hline$Q_{D 3}$ & $\left(\tilde{N}_{i}, \tilde{M}_{i}\right)$ & $g_{s}$ & $W_{0}$ & $\left|W_{0}\right|$ & $\left|F_{\text {inst }}\right| /|F|$ & $\max _{i}\left(\left|F_{\text {inst }}^{i}\right|\right) /|F|$ \\
\hline 19 & $(1,-3,0,0,0,0,2,-1)$ & 0.39 & $618 .+727.3 i$ & 954.4 & 0.0744 & 0.00976 \\
\hline 14 & $(1,1,1,1,3,0,0,0)$ & 0.8 & $-41.33-71.58 i$ & 82.66 & 0.731 & 0.0592 \\
\hline 14 & $(1,0,0,1,1,-3,1,0)$ & 0.75 & $-9.465+53.2 i$ & 54.03 & 0.723 & 0.051 \\
\hline 15 & $(2,0,2,1,0,0,-1,0)$ & 0.77 & $49.89+25.11 i$ & 55.85 & 0.0114 & 0.00387 \\
\hline 15 & $(1,2,1,1,1,2,-1,0)$ & 0.45 & $-30.06-20.59 i$ & 36.44 & 0.788 & 0.0968 \\
\hline 18 & $(1,2,0,2,2,2,0,-1)$ & 0.29 & $-120.3-38.86 i$ & 126.5 & 0.014 & 0.0257 \\
\hline
\end{tabular}

Table 2. Examples of solutions which satisfy $0<g_{s}<1$ and obey $\left|F_{\text {inst }}\right| /|F|<1$, $\max _{i}\left(\left|F_{\text {inst }}^{i}\right|\right) /|F|<0.1$. Here we show the respective size of their instanton contributions. Again note that the third and respectively seventh entry for the flux configuration denote the entries for $\tilde{N}_{2}=\tilde{N}_{3}$ respectively $\tilde{M}_{2}=\tilde{M}_{3}$ which are chosen to be the same as described in the main text. $F_{\text {inst }}^{i}$ denotes the leading order contribution in $F_{\text {inst }}$ in a given $u_{i}$.

figure 3 the distribution in the $\left(g_{s},\left|W_{0}\right|\right)$-plane after restricting to the fundamental domain: most of the points (shown in gray) correspond to points with numerically large instanton contributions, whereas for blue and red points the instanton contributions are numerically small (satisfying $\left|F_{\text {inst }}\right| /|F|<0.1$ and $\max _{i}\left(\left|F_{\text {inst }}^{i}\right|\right) /|F|<0.1$ ) taking instanton corrections up-to order 2 (blue) and order 10 (red) into account. This numerical cut is chosen as in [27] to guarantee that the instanton contributions to the (pre-)potential are at least an order of magnitude smaller compared to the overall prepotential.

The distribution of minima (again before invoking the small contributions of the instantons) in the fundamental domain is shown in figure 4.

Finally, in table 1 and 2, we display some minima with 'small' worldsheet instanton contributions. For illustrative purposes we show a variety of solutions with different tadpoles, flux configurations and different values for the stabilised moduli and flux superpotential, requiring not necessarily the above smallness criteria applied in the distribution plots. As explained above this is a necessary requirement for retaining the minimum obtained from the classical terms also in the presence of the instanton corrections.

In order to have a consistent superspace derivative expansion in the $4 \mathrm{D}$ effective field theory, one requires $\mathcal{V} \gg\left|W_{0}\right|^{3}[61]$. This implies that the Kähler moduli have to be stabilised such that $\mathcal{V} \gg 10^{3}$, respectively, $\mathcal{V} \gg 10^{6}$ depending on $\left|W_{0}\right|$. 


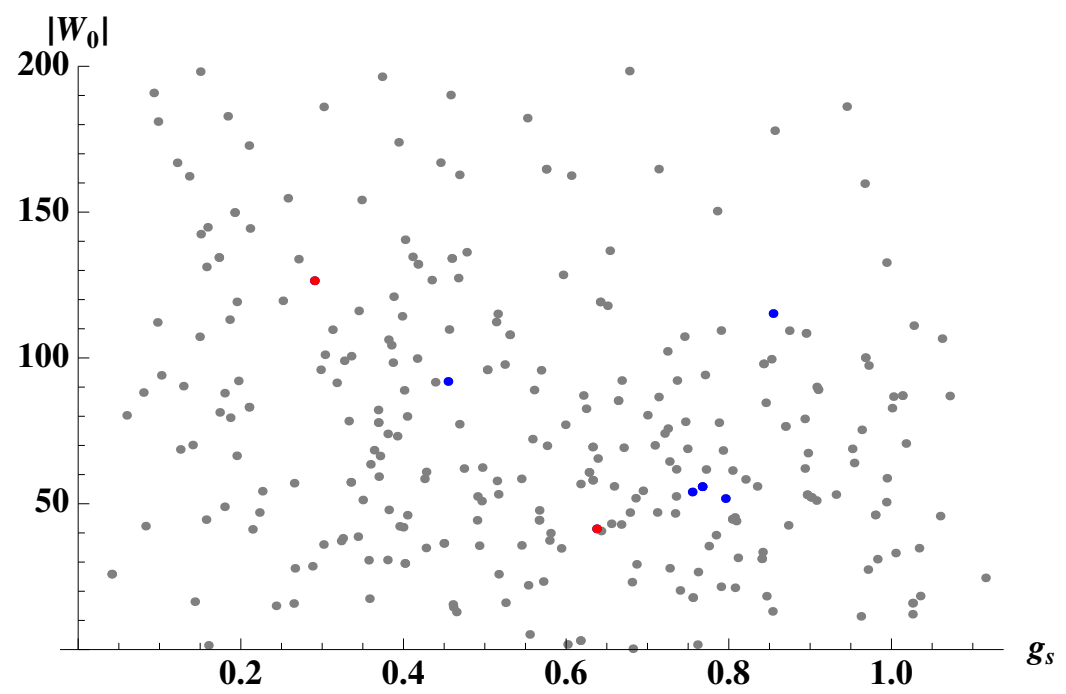

Figure 3. Distribution of flux solutions in the $\left(g_{s},\left|W_{0}\right|\right)$-plane in the fundamental domain after performing appropriate $\mathrm{SL}(2, \mathbb{Z})$ transformations.
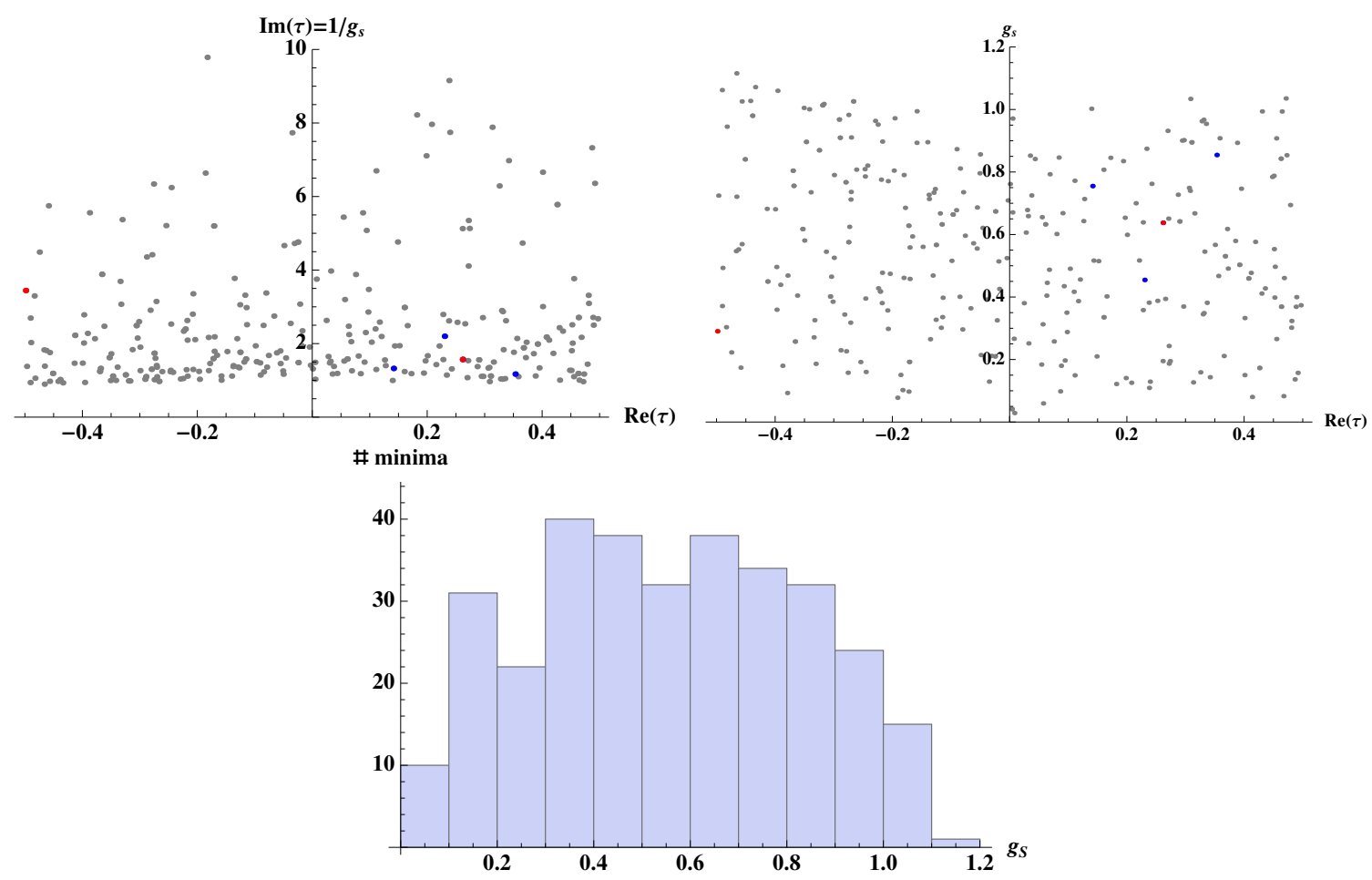

Figure 4. Distribution of solutions in the fundamental domain of $\tau$, i.e. in the $\left(\operatorname{Re}[\tau], \operatorname{Im}[\tau]=1 / g_{s}\right)$ plane (top left). Next to it (top right) we show the distribution in the $\left(\operatorname{Re}[\tau], 1 / \operatorname{Im}[\tau]=g_{s}\right)$ plane. The bottom shows the distribution of minima with respect to values of $g_{s}$ before restricting to small worldsheet instanton corrections. Gray points correspond to points with large instanton corrections, blue and red points have small contributions from instantons taking corrections up to order 2 and 10 into account. 

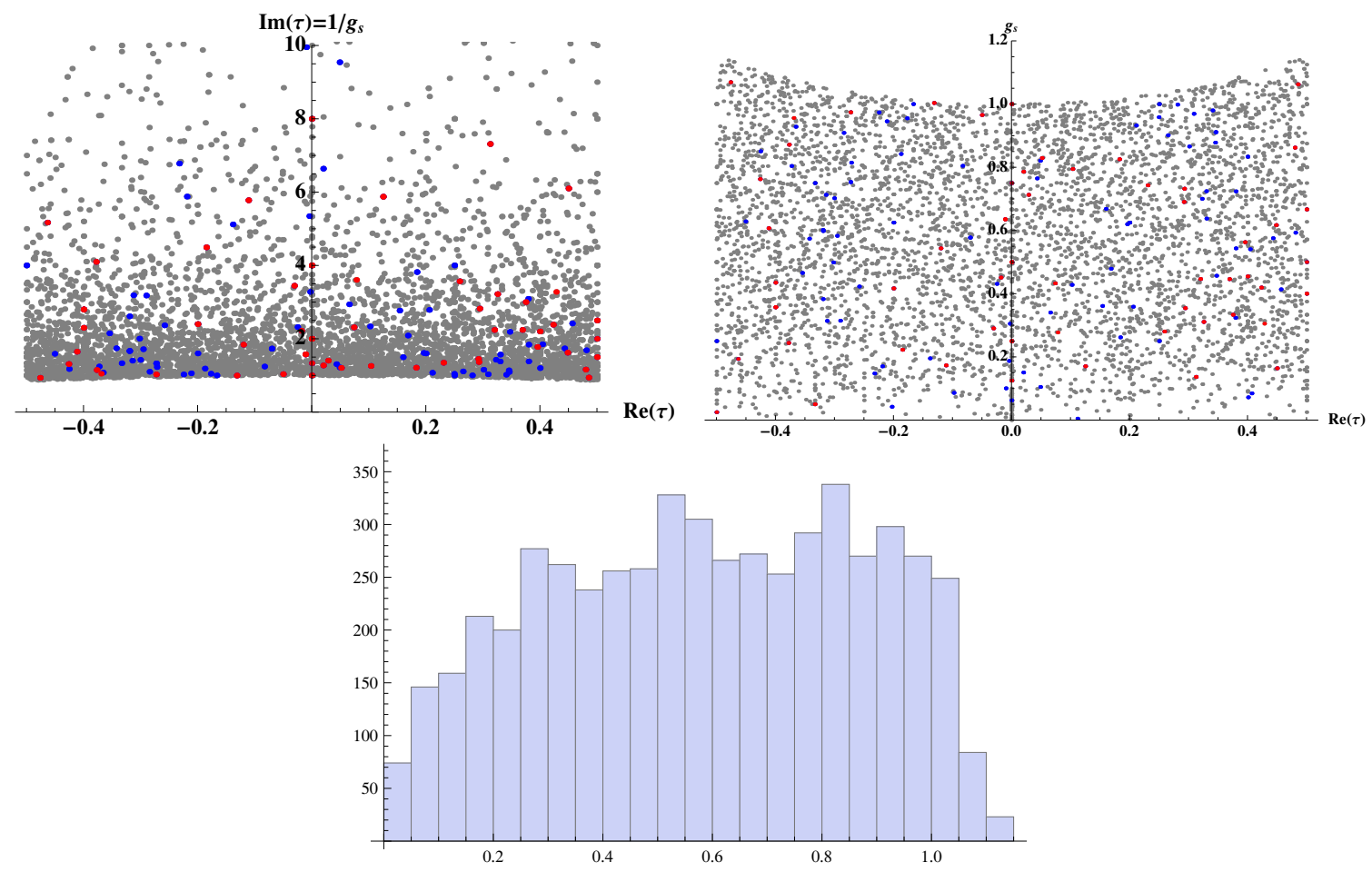

Figure 5. The same distributions as before but for $\mathbb{P}_{1,1,1,6,9}$. Distribution of solutions in the fundamental domain of $\tau$, i.e. in the $\left(\operatorname{Re}[\tau], \operatorname{Im}[\tau]=1 / g_{s}\right)$ plane (top). In the middle we show the distribution in the $\left(\operatorname{Re}[\tau], 1 / \operatorname{Im}[\tau]=g_{s}\right)$ plane. The bottom shows the distribution of minima with respect to values of $g_{S}$ before restricting to points with small worldsheet instanton corrections. Gray points correspond to points with large instanton corrections, blue and red points have instanton contributions that are small taking corrections up to order 2 and 14 into account (again requiring $\left|F_{\text {inst }}\right| /|F|<0.1$ and $\left.\max _{i}\left(\left|F_{\text {inst }}^{i}\right|\right) /|F|<0.1\right)$.

To compare our results to previous analysis, we show in figure 5 the corresponding distributions for $\mathbb{P}_{[1,1,1,6,9]}^{4}[18]$. Note that the gray points in figures 3 and 4 are not solutions of $D W=0$ when $W$ is the full type IIB superpotential. On the other hand, they satisfy $D \hat{W}=0$, where $\hat{W}$ is the cubic polynomial in the $u_{i}$ arising from the prepotential without instanton corrections as in (2.28). We can consider $\hat{W}$ as the superpotential for a toy model. From the figures 3 and 4 , we see that in this toy model we find results consistent with a uniform distribution of $W_{0}$ and $g_{s}$.

\subsection{Kähler moduli stabilisation}

As we have seen in the previous section, the dilaton and the complex structure moduli can be fixed supersymmetrically at semi-classical level by turning on quantised background fluxes. For concreteness, we shall focus on the flux vacuum related to the last line in table 1: the string coupling is in the perturbative regime, $g_{s}=0.29$, the VEV of the flux superpotential is $\left|W_{0}\right|=126.5$ and the D3-charge of the flux is $Q_{D 3}=18$, leaving space for $N_{D 3} \leq 2 \mathrm{D} 3$-branes on top of the $\mathrm{dP}_{6}$ singularities. 
Definition of the Kähler moduli. Due to the no-scale property of the type IIB Kähler potential for the Kähler moduli $T_{i}, i=1, \ldots, h^{1,1}\left(X_{3}\right), K_{T-\text { moduli }}=-2 \ln \mathcal{V}$, the fluxes do not induce any tree-level F-term potential for the $T$-moduli. The Kähler moduli can however be lifted by either perturbative or non-perturbative effects. A general strategy to lift these remaining flat directions in a way compatible with the presence of chirality has been described in $[15,16]$. Here we shall simply apply the same stabilisation scheme to our particular example.

The total number of Kähler moduli is $h^{1,1}\left(X_{3}\right)=4$. Three of them are even under the orientifold and are defined as:

$$
T_{b}=\tau_{b}+\mathrm{i}\left(\int_{\mathcal{D}_{b}} C_{4}\right), \quad T_{s}=\tau_{s}+\mathrm{i}\left(\int_{\mathcal{D}_{s}} C_{4}\right), \quad T_{\mathrm{vs}}=\tau_{\mathrm{vs}}+\mathrm{i}\left(\int_{\mathcal{D}_{q_{1}}+\mathcal{D}_{q_{2}}} C_{4}\right),
$$

where the visible sector four-cycle volume is $\tau_{\mathrm{vs}}=\tau_{q_{1}}+\tau_{q_{2}}$. On the other hand, the orientifold odd Kähler modulus is defined as [62]:

$$
G=b+\mathrm{i} c \equiv\left(\int_{\mathcal{D}_{q_{1}}-\mathcal{D}_{q_{2}}} B_{2}\right)+\mathrm{i}\left(\int_{\mathcal{D}_{q_{1}}-\mathcal{D}_{q_{2}}} C_{2}\right)
$$

Leading order stabilisation. The potential for the Kähler moduli can be organised in an expansion in inverse powers of the overall $\mathrm{CY}$ volume $\mathcal{V}$. As we shall see, the leading order contribution comes from D-terms which depend on the Kähler moduli because gauge fluxes induce $T$-dependent FI-terms.

In addition to the anomalous $\mathrm{U}(1)$ living on the stack of D7-branes on top of the O7plane, there are two additional anomalous $\mathrm{U}(1)$ symmetries belonging to the gauge theory located at the $\mathrm{dP}_{6}$ singularity. ${ }^{22}$ Thus the total D-term potential looks like:

$$
V_{D}=V_{D}^{\text {quiver }}+V_{D}^{\text {bulk }}
$$

where:

$$
V_{D}^{\text {quiver }}=\frac{1}{\operatorname{Re}\left(f_{D 3_{1}}\right)}\left(\sum_{i} q_{D 3_{1} i}\left|C_{i}\right|^{2}-\xi_{D 3_{1}}\right)^{2}+\frac{1}{\operatorname{Re}\left(f_{D 3_{2}}\right)}\left(\sum_{i} q_{D 3_{2} i}\left|C_{i}\right|^{2}-\xi_{D 3_{2}}\right)^{2},
$$

and:

$$
V_{D}^{\text {bulk }}=\frac{1}{\operatorname{Re}\left(f_{D 7}\right)}\left(\sum_{i} q_{D 7 i}\left|\phi_{i}\right|^{2}-\xi_{D 7}\right)^{2}
$$

In the previous expressions, the gauge kinetic functions are given by:

$$
f_{D 3_{1}}=\tau+q_{D 3_{1}} T_{\mathrm{vs}}, \quad f_{D 3_{2}}=\tau+q_{D 3_{2}} G \quad \text { and } \quad f_{D 7}=T_{b}-T_{s}+k \tau
$$

where $q_{D 3_{i}}$ are the $\mathrm{U}(1)$ charges of $T_{\mathrm{vs}}$ and $G$ while $k$ is a parameter which depends on $\mathcal{F}_{D 7}$. The $C_{i}$ and $\phi_{i}$ are canonically normalised matter fields with $\mathrm{U}(1)$ charges $q_{D 3_{1} i}, q_{D 3_{2} i}$

\footnotetext{
${ }^{22}$ The gauge theory associated to any $\mathrm{dP}_{n}$ singularity, with $n=0,1, \ldots, 8$, gives always rise to two anomalous U(1)'s.
} 
and $q_{D 7 i}$ living respectively on the fractional D3- and D7-branes. ${ }^{23}$ The FI-terms $\xi_{D 3_{1}}$ and $\xi_{D 3_{2}}$ read [63]:

$$
\xi_{D 3_{1}}=4 q_{D 3_{1}} \frac{\tau_{\mathrm{vs}}}{\mathcal{V}} \quad \text { and } \quad \xi_{D 3_{2}}=4 q_{D 3_{2}} \frac{b}{\mathcal{V}}
$$

while $\xi_{D 7}$ is given in (3.31).

One can easily see that the D-term potential scales as $V_{D}^{\text {quiver }} \sim V_{D}^{\text {bulk }} \sim \mathcal{O}\left(1 / \mathcal{V}^{2}\right)$. This is of the same order as the flux-generated F-term potential used to fix $\tau$ and all the complex structure moduli:

$$
V_{F}^{\text {flux }} \simeq \frac{1}{\mathcal{V}^{2}}\left(\left|D_{\tau} W\right|^{2}+\left|D_{u} W\right|^{2}\right) .
$$

In order to have a consistent stabilisation procedure, we have therefore to set $V_{D}=0$ so that supersymmetry is preserved at this order of expansion in inverse powers of $\mathcal{V}$. This condition gives:

$$
\xi_{D 3_{1}}=\sum_{i} q_{D 3_{1} i}\left|C_{i}\right|^{2}, \quad \xi_{D 3_{2}}=\sum_{i} q_{D 3_{2} i}\left|C_{i}\right|^{2} \quad \text { and } \quad \xi_{D 7}=\sum_{i} q_{D 7 i}\left|\phi_{i}\right|^{2} .
$$

These relations fix only three combinations of closed and open string moduli, corresponding to the combinations of axions which get eaten up by the anomalous $\mathrm{U}(1) \mathrm{s}$ via the Stückelberg mechanism. The mass of each Abelian gauge boson is proportional to the open and closed string axion decay constants $f_{\mathrm{ax}, j}^{\mathrm{op}}$ and $f_{\mathrm{ax}, j}^{\mathrm{cl}}[64]$ :

$$
M_{\mathrm{U}(1), j}^{2} \simeq \frac{1}{\operatorname{Re}\left(f_{j}\right)}\left[\left(f_{\mathrm{ax}, j}^{\mathrm{op}}\right)^{2}+\left(f_{\mathrm{ax}, j}^{\mathrm{cl}}\right)^{2}\right]
$$

where:

$$
\left(f_{\mathrm{ax}, j}^{\mathrm{op}}\right)^{2} \simeq \xi_{j} \quad \text { and } \quad\left(f_{\mathrm{ax}, j}^{\mathrm{cl}}\right)^{2} \simeq \frac{\partial^{2} K}{\partial T_{j}^{2}}
$$

For the anomalous $\mathrm{U}(1)$ on the D7-stack in the bulk, we have:

$$
\left(f_{\mathrm{ax}, D 7}^{\mathrm{op}}\right)^{2} \simeq \xi_{D 7} \simeq \frac{1}{\tau_{b}} \gg\left(f_{\mathrm{ax}, D 7}^{\mathrm{cl}}\right)^{2} \simeq \frac{\partial^{2} K}{\partial T_{b}^{2}} \simeq \frac{1}{\tau_{b}^{2}} \quad \text { for } \quad \tau_{b} \sim \mathcal{V}^{2 / 3} \gg 1,
$$

implying that the combination of moduli fixed by D-terms is mostly given by open string modes. We can therefore consider $\tau_{b}$, or equivalently $\mathcal{V}$, as a flat direction at this level of approximation. As can be seen from (4.12), the anomalous $\mathrm{U}(1)$ develops a mass of the order of the Kaluza-Klein scale: $M_{\mathrm{U}(1), D 7} \sim M_{P} / \tau_{b} \sim M_{P} / \mathcal{V}^{2 / 3} \sim M_{K K}$.

On the other hand, the Abelian gauge bosons at the $\mathrm{dP}_{6}$ singularity become massive by eating up the closed string axions since:

$$
K \supset \frac{\left(T_{\mathrm{vs}}+\bar{T}_{\mathrm{vs}}\right)^{2}}{\mathcal{V}} \Rightarrow\left(f_{\mathrm{ax}, D 3_{1}}^{\mathrm{op}}\right)^{2} \simeq \frac{\tau_{\mathrm{vs}}}{\mathcal{V}} \ll\left(f_{\mathrm{ax}, \mathrm{D} 3_{1}}^{\mathrm{cl}}\right)^{2} \simeq \frac{\partial^{2} K}{\partial T_{\mathrm{vs}}^{2}} \simeq \frac{1}{\mathcal{V}} \quad \text { for } \quad \tau_{\mathrm{vs}} \ll 1,
$$

and similarly for the second $\mathrm{U}(1)$. Hence the D-terms from the quiver construction fix $\tau_{\mathrm{vs}}$ and $b$. The corresponding axions get eaten up by the two anomalous $\mathrm{U}(1) \mathrm{s}$ which from (4.12) acquire a mass of the order of the string scale: $M_{\mathrm{U}(1), D 3} \sim M_{P} / \mathcal{V}^{1 / 2} \sim M_{s}$. Similarly to $\tau_{b}$, the matter fields $\left|C_{i}\right|$ remain massless at this level of approximation.

\footnotetext{
${ }^{23}$ Note that we denoted the fields $X, Y, Z$ of section 3.2 as $C_{i}$, while the $\phi_{i}$ are fields in the anti-symmetric representation of $\mathrm{U}(4)$, cf. (56).
} 
Subleading order stabilisation. In order to fix $T_{b}, T_{s}$ and $\left|C_{i}\right|$ we have to consider subleading F-term contributions.

Visible sector matter fields. Focusing on the stabilisation of the visible sector matter fields, we have (we restrict to the case of just one open string field $C$ where we set the VEV of its phase to zero without loss of generality $\left.{ }^{24}\right)$ :

$$
\begin{aligned}
V_{F}(|C|) & =c_{2} m_{0}^{2}|C|^{2}+c_{3} A|C|^{3}+c_{4} \lambda|C|^{4}+\mathcal{O}\left(|C|^{5}\right)-d_{4} \frac{\tau_{\mathrm{vs}}^{2}}{\mathcal{V}^{3}}\left[1+\mathcal{O}\left(\frac{1}{\mathcal{V}}\right)\right] \\
& \simeq c_{2} \frac{|C|^{2}}{\mathcal{V}^{\alpha_{2}}}+c_{3} \frac{|C|^{3}}{\mathcal{V}^{\alpha_{3}}}+\left(\frac{c_{4}}{\mathcal{V}^{\alpha_{4}}}-\frac{d_{4}}{\mathcal{V}}\right)|C|^{4},
\end{aligned}
$$

where the first three terms come from expanding the F-term scalar potential in powers of $|C|$ up to fourth order, while the last term comes from the breaking of the no-scale structure by $\tau_{\mathrm{vs}}$ which we wrote in terms of $|C|$ using the first relation in (4.11). In more detail, the quadratic term is proportional to the soft scalar mass of $|C|$ which we have parametrised as $m_{0}^{2} \simeq \mathcal{V}^{-\alpha_{2}}$, the cubic term is proportional to the soft $A$-term parametrised as $A \simeq \mathcal{V}^{-\alpha_{3}}$, while the quartic term is proportional to the coupling $\lambda \simeq \mathcal{V}^{-\alpha_{4}}$.

Depending on the sign of $c_{2}$, the VEV of $|C|$ can be zero or non-vanishing. If $c_{2}>0$, then $|C|=0$, implying, by (4.11), $\xi_{D 3, i}=0 \forall i=1,2$ which fixes $\tau_{\mathrm{vs}}=b=0$. This ensures that the $\mathrm{dP}_{6}$ divisor supporting the visible sector is really collapsed to zero size.

If instead the matter field $|C|$ develops a tachyonic mass from supersymmetry breaking, i.e. $c_{2}<0$, then, depending on the signs of the different coefficients in (4.15), $|C|$ can develop a non-zero $\mathrm{VEV}$. In this case, one may worry that $\tau_{\mathrm{vs}}$ gets fixed at values larger than the string scale, so resolving the $\mathrm{dP}_{6}$ singularity. However this is not the case. In fact, for models with just fractional D3-branes, the visible sector is sequestered from supersymmetry breaking, leading to $\alpha_{3}=2, \alpha_{4}=1$ and either $\alpha_{2}=3$ or $\alpha_{2}=4$, depending on the modulidependence of the Kähler metric for matter fields [65]. Thus $|C| \simeq M_{P} / \mathcal{V}^{\alpha_{2}-2}$, implying from (4.11) that $\tau_{\mathrm{vs}} \simeq \mathcal{V}^{5-2 \alpha_{2}}$, with a similar relation holding for $b$. We then see that for $\alpha_{2}=3 \tau_{\mathrm{vs}} \sim \mathcal{V}^{-1} \ll 1$, while for $\alpha_{2}=4 \tau_{\mathrm{vs}} \sim \mathcal{V}^{-3} \ll 1$, and so in both cases the $\mathrm{dP}_{6}$ divisor is still in the singular regime.

An interesting implication of this case is that the phase $\theta$ of $C=|C| e^{\mathrm{i} \theta}$ can behave as the QCD axion for $\alpha=3$. In fact, its decay constant $f_{\mathrm{ax}}^{\mathrm{op}}$ is set by the above VEV of $|C|$ which can take two different values (using $\mathcal{V} \sim 10^{8}$ as derived below):

$$
f_{\mathrm{ax}}^{\mathrm{op}} \simeq \frac{M_{P}}{\mathcal{V}} \simeq 10^{10} \mathrm{GeV} \quad \text { for } \quad \alpha_{2}=3 \quad \text { and } \quad f_{\mathrm{ax}}^{\mathrm{op}} \simeq \frac{M_{P}}{\mathcal{V}^{2}} \simeq 100 \mathrm{GeV} \quad \text { for } \quad \alpha_{2}=4 .
$$

The value of $f_{\mathrm{ax}}^{\mathrm{op}}$ for $\alpha_{2}=3$ lies inside the phenomenologically allowed window for the QCD axion: $10^{9} \mathrm{GeV} \lesssim f_{\mathrm{QCD}} \lesssim 10^{12} \mathrm{GeV}$ while the case with $\alpha_{2}=4$ seems to be ruled out. Note that $C$ has to be a SM singlet due to the large value of its VEV. Moreover, there are two anomalous $\mathrm{U}(1) \mathrm{s}$ at the $\mathrm{dP}_{6}$ singularity, and so only one combination of open string axions can play the rôle of the QCD axion and get massive by QCD instantons whereas the other combination would remain massless.

\footnotetext{
${ }^{24} \mathrm{~A}$ different value of the phase could change the sign of $c_{3}$, for example, but given that we are not working out the exact sign of $c_{3}$, the exact value of the phase is irrelevant for our conclusions.
} 
In the presence of flavour D7-branes, threshold effects de-sequester the visible sector inducing soft terms of order the gravitino mass, and so $\alpha_{2}=2$. In this case $c_{2}$ has to be positive otherwise $\tau_{\mathrm{vs}}$ would develop a VEV of order unity, thus resolving the $\mathrm{dP}_{6}$ singularity. The matter field $|C|$ would therefore have a vanishing VEV which could however become non-zero via RG running below the string scale. $\tau_{\mathrm{vs}}$ would then develop a very small VEV compatible with the singular regime and one could obtain a viable QCD axion from the phase of a matter field analogous to the situation described above. A similar mechanism could help to have a viable QCD axion also in the case with just fractional D3-branes and $\alpha_{2}=4$.

Kähler moduli in the geometric regime. The scalar potential for the Kähler moduli $T_{s}$ and $T_{b}$ receives three different leading order contributions: $V_{\mathrm{np}}$ from non-perturbative superpotential effects, $V_{\alpha^{\prime}}$ from perturbative corrections to the Kähler potential and soft terms $V_{\text {soft }}$ from matter fields living on bulk D7-branes.

The expression for $V_{\mathrm{np}}$ is [5]:

$$
V_{\mathrm{np}}=\frac{8}{3 \lambda}\left(a_{s} A_{s}\right)^{2} \sqrt{\tau_{s}} \frac{e^{-2 a_{s} \tau_{s}}}{\mathcal{V}}-4 a_{s} A_{s}\left|W_{0}\right| \tau_{s} \frac{e^{-a_{s} \tau_{s}}}{\mathcal{V}^{2}}
$$

where we have already minimised with respect to the $T_{s}$-axion and we have traded $\tau_{b}$ for $\mathcal{V} \simeq \lambda \tau_{b}^{3 / 2}$. This potential is generated by an E3-instanton wrapping $\mathcal{D}_{s}$ with $a_{s}=2 \pi$, while the prefactor $A_{s}$ is function of the stabilised complex structure moduli as well as of potential open string moduli, whose exact structure is hard to compute in practice. This instanton contributes to the superpotential if the $B$-field is chosen as in (3.25). If one instead performs the flux choice (3.24), gaugino condensation in the $\mathrm{SO}(8)$ pure super Yang-Mills theory on the bulk D7-branes gives rise to a tiny contribution proportional to $e^{-\frac{\pi}{3} \tau_{b}}$ which can be neglected. Moreover, a possible contribution from a rank-two instanton on $\mathcal{D}_{s}$ would also be negligible for $\tau_{s}>1$.

The contribution $V_{\alpha^{\prime}}$ takes the form [66]:

$$
V_{\alpha^{\prime}}=\frac{3}{4} \frac{\zeta\left|W_{0}\right|^{2}}{g_{s}^{3 / 2} \mathcal{V}^{3}},
$$

where $\zeta=-\chi\left(X_{3}\right) \zeta(3) /\left[2(2 \pi)^{3}\right] \simeq 0.32$ using (2.20) for the Euler number of $X_{3}$. Further perturbative corrections arise due to string loop effects $[67,68] .{ }^{25}$ Because of the 'extended no-scale structure' [74, 75], these corrections are negligible since they scale as $\mathcal{V}^{-10 / 3}$ whereas $V_{\alpha^{\prime}}$ behaves as $\mathcal{V}^{-3}$.

The last contribution $V_{\text {soft }}$ reads:

$$
V_{\text {soft }}=c \frac{\left|W_{0}\right|^{2}}{\mathcal{V}^{2}\left[\ln \left(\mathcal{V} /\left|W_{0}\right|\right)\right]^{2}}|\phi|^{2},
$$

where the soft scalar mass for the matter field $\phi$ living in the bulk scales as the gravitino mass $m_{3 / 2}=e^{K / 2}|W| \simeq\left|W_{0}\right| / \mathcal{V}$ suppressed by a factor of order $\ln \left(M_{P} / m_{3 / 2}\right)[76,77](c$ is

\footnotetext{
${ }^{25}$ For recent activity on stringy perturbative corrections see [69-73].
} 
an $\mathcal{O}(1)$ coefficient). Employing the last relation in (4.11) one obtains:

$$
V_{\text {soft }}=p \frac{\left|W_{0}\right|^{2}}{\mathcal{V}^{8 / 3}\left[\ln \left(\mathcal{V} /\left|W_{0}\right|\right)\right]^{2}}, \quad \text { with } \quad p=\frac{c I_{\mathrm{U}(4)}}{2^{1 / 3} q_{D 7}},
$$

with $I_{\mathrm{U}(4)}$ given in (3.28).

Minimising the total F-term potential $V_{F}^{\text {tot }}=V_{\text {np }}+V_{\alpha^{\prime}}+V_{\text {soft }}$ with respect to $\tau_{s}$ in the limit $\epsilon \equiv 1 /\left(a_{s} \tau_{s}\right) \ll 1$, we obtain:

$$
e^{-a_{s} \tau_{s}} \simeq \frac{3 \lambda \sqrt{\tau_{s}}}{4 a_{s} A_{s}} \frac{\left|W_{0}\right|}{\mathcal{V}} \quad \Rightarrow \quad \tau_{s}(\mathcal{V}) \simeq \frac{1}{a_{s}} \ln \left(\frac{\mathcal{V}}{\left|W_{0}\right|}\right)
$$

Plugging this result into $V_{F}^{\text {tot }}$, we find:

$$
V \simeq \frac{\left|W_{0}\right|^{2}}{\mathcal{V}^{3}}\left[\frac{3 \zeta}{4 g_{s}^{3 / 2}}-\frac{3 \lambda}{2} \tau_{s}(\mathcal{V})^{3 / 2}+p \frac{\mathcal{V}^{1 / 3}}{\left[a_{s} \tau_{s}(\mathcal{V})\right]^{2}}\right] .
$$

The minimisation with respect to $\mathcal{V}$ then gives:

$$
\frac{3 \zeta}{4 g_{s}^{3 / 2}} \simeq \frac{3 \lambda}{2} \tau_{s}(\mathcal{V})^{3 / 2}\left(1-\frac{1}{2 a_{s} \tau_{s}(\mathcal{V})}\right)-\frac{8}{9} p \frac{\mathcal{V}^{1 / 3}}{\left[a_{s} \tau_{s}(\mathcal{V})\right]^{2}}
$$

Substituting this result in (4.21), the leading order contribution to the vacuum energy becomes:

$$
\left\langle V_{F}^{\text {tot }}\right\rangle \simeq \frac{\left|W_{0}\right|^{2}}{\langle\mathcal{V}\rangle^{3}} \sqrt{a_{s} \tau_{s}(\mathcal{V})}\left[-\frac{3 \lambda}{4 a_{s}^{3 / 2}}+\frac{p}{9} \frac{\langle\mathcal{V}\rangle^{1 / 3}}{\left[a_{s} \tau_{s}(\mathcal{V})\right]^{5 / 2}}\right]
$$

Setting this expression equal to zero and plugging the result for $p$ back in (4.22), we find:

$$
\frac{3 \zeta}{4 g_{s}^{3 / 2}} \simeq \frac{3 \lambda}{2} \tau_{s}^{3 / 2}(1+\mathcal{O}(\epsilon))
$$

For $g_{s}=0.29,\left|W_{0}\right|=126.5$ and $\lambda=\frac{1}{3} \sqrt{\frac{2}{3}}$ from (3.7), (4.24) yields $\tau_{s} \simeq 2.42$ which fixes the divisor $\mathcal{D}_{s}$ supporting the E3-instanton in the geometric regime above the string scale. Setting $a_{s}=2 \pi$, the relation (4.20) then yields $\mathcal{V} \simeq 2.55 \cdot 10^{7} / A_{s}$.

We compute the range for the coefficient $A_{s}$ by imposing consistency with TeV-scale supersymmetry. ${ }^{26}$ If there are no flavour branes and the visible sector is sequestered, the gaugino masses scale as [65]:

$$
M_{1 / 2}=\frac{3 \gamma \zeta e^{K_{\mathrm{cs}} / 2}}{4 \sqrt{2} g_{s}} \frac{\left|W_{0}\right| M_{P}}{\mathcal{V}^{2}} \simeq 36.5 \mathrm{TeV} \gamma A_{s}^{2}
$$

where $\gamma$ is an $\mathcal{O}(1)$ function of the complex structure moduli which determines the shift of the dilaton VEV due to $\alpha^{\prime}$ effects, and $e^{K_{\mathrm{cs}}} \simeq 1 / 55.9$ by direct computation in our explicit example. If $\gamma=1$ and $A_{s}=0.2$, one obtains $M_{1 / 2} \simeq 1.5 \mathrm{TeV}$. The $\mathrm{CY}$ volume is then $\mathcal{V} \simeq 1.3 \cdot 10^{8}$ while the parameter $p$ becomes $p \simeq 0.2$. As can be seen from (4.19), $p$ depends

\footnotetext{
${ }^{26}$ Note that the coefficient $A_{s}$ depends on open and closed strings (see [78] for the dependence of the prefactor on D3 brane moduli). An explicit stabilisation of this quantity in this range for TeV scale supersymmetry is an additional constraint on open/closed string moduli stabilisation.
} 
just on the flux coefficient $n_{b}$ introduced in (3.26), the $\mathrm{U}(1)$ charge $q_{D 7}$ and the parameter $c$, and so we do not have enough freedom to tune the cosmological constant. This was expected from the beginning since we chose a particular value of the flux superpotential $\left|W_{0}\right|$. However, $p$ turns out to be of order unity, implying that in order to find a solution with vanishing cosmological constant one has to vary $\left|W_{0}\right|$ around the value considered in this example, $\left|W_{0}\right|=126.5$.

If the visible sector is not sequestered from supersymmetry breaking, then the soft terms become of the order of the gravitino mass and a larger value of $\mathcal{V}$ is needed, resulting in a larger tuning of $A_{s}$. We shall therefore focus on the sequestered case where the string scale turns out to be $M_{s}=\sqrt{\pi} g_{s}^{1 / 4} M_{P} / \sqrt{\mathcal{V}} \simeq 2 \cdot 10^{14} \mathrm{GeV}$. The unification scale is instead set by the winding scale $M_{W} \simeq M_{s} \mathcal{V}^{1 / 6} \simeq 5 \cdot 10^{15} \mathrm{GeV}$. The gravitino mass is intermediate, $m_{3 / 2}=e^{K_{\mathrm{cs}} / 2} \sqrt{g_{s} / 2}\left|W_{0}\right| M_{P} / \mathcal{V} \simeq 6 \cdot 10^{10} \mathrm{GeV}$, whereas the moduli masses scale as:

$$
m_{\tau_{s}} \simeq m_{3 / 2} \ln \left(M_{P} / m_{3 / 2}\right) \simeq 1 \cdot 10^{12} \mathrm{GeV} \quad \text { and } \quad m_{\mathcal{V}} \simeq \frac{m_{3 / 2}}{\sqrt{\mathcal{V}}} \simeq 5 \cdot 10^{6} \mathrm{GeV}
$$

showing that there is no cosmological moduli problem. Recall that the cosmological moduli problem places a lower bound on the moduli masses $\left(m_{\text {mod }} \gtrsim \mathcal{O}(10) \mathrm{TeV}\right.$, cf. [79-81]). Both $\tau_{\mathrm{vs}}$ and $b$ get a mass of order the string scale, justifying the fact that they can be integrated out. On the other hand, the complex structure moduli and the dilaton develop a mass of order $m_{3 / 2}$. They can however still be integrated out at leading order since they are decoupled from the $T$-moduli (at least at classical level the Kähler potential factorises). As far as the axions are concerned, the $T_{s}$-axion gets a mass of order $m_{\tau_{s}}$ while the volume axion is massless at this level of approximation. It will only get a tiny mass by subleading non-perturbative effects which scale as $e^{-\mathcal{V}^{2 / 3}} \ll 1$. This bulk axion behaves as dark radiation $[82,83]$ and gets produced by the decay of the light volume modulus which drives reheating after the end of inflation.

\section{Summary and conclusions}

The general ideas regarding realistic local string models and moduli stabilisation are based on several reasonable assumptions but it is important to substantiate them with concrete realisations in order to put those proposals on firmer grounds. We have been able to combine several ideas that have been implemented independently before - chiral matter at branes at singularities, fluxes to stabilise complex structure moduli and quantum effects to stabilise Kähler moduli — in one single framework. This is a technically challenging achievement and represents a clear step forward towards the explicit construction of fully realistic models. It is very encouraging that properties such as the unification and the supersymmetry breaking scale are obtained at realistic values while at the same time allowing for de Sitter vacua.

The procedure followed here has been systematic:

- First of all, we find appropriate CY manifolds that satisfy the phenomenological requirements: having at least four Kähler moduli, two rigid cycles mapped into each 
other by orientifold involution to guarantee a visible sector with $\mathrm{U}(n)$ gauge symmetries, an extra rigid cycle in the geometric regime for non-perturbative moduli stabilisation and an extra cycle which controls the overall volume. Furthermore, these varieties admit suitable discrete symmetries to effectively reduce the number of complex structure moduli.

- We identify the orientifold action and its associated O-planes. We determine the structure and location of D-branes wrapping different cycles in order to satisfy all consistency conditions. In addition, we make sure that at least one cycle allows a non-zero contribution to the non-perturbative superpotential.

- In order to stabilise the dilaton and the complex structure moduli, we turn on arbitrary fluxes on three-cycles invariant under the discrete symmetry. This reduces the complex structure stabilisation to a manageable problem.

- All geometric moduli are stabilised in a self-consistent manner. Assuming that the overall volume is large enough to justify a $1 / \mathcal{V}$ expansion, we start with the dominant terms of order $1 / \mathcal{V}^{2}$. These are the F-terms for the dilaton and the complex structure moduli, as well as the D-terms. Since all of them are positive definite, they minimise the overall scalar potential at zero value to leading order in $1 / \mathcal{V}$. The F-terms fix the dilaton and complex structure moduli. The D-terms induced by anomalous U(1)s, both, at the visible and hidden sector, fix the value of matter fields in the hidden sector and blow-up modes in the visible sector. The corresponding axion fields get eaten by the Abelian gauge bosons as in the standard Stückelberg mechanism. At next order, i.e. $1 / \mathcal{V}^{3}$, the geometric Kähler moduli are stabilised as in the standard LVS scenario, giving rise to an exponentially large volume and supersymmetry breaking.

Besides the three 'standard terms' that determine the large volume minimum, there are non-vanishing F-terms of bulk matter fields residing on the brane wrapping the large cycle. These additional contributions are induced by D-term stabilisation and supersymmetry breaking. They add a positive term which uplifts the value of the minimum.

Depending on the VEV of the flux superpotential $\left|W_{0}\right|$, the minimum can be AdS, dS or Minkowski. We select the value (in the dense uniform range) that gives approximately Minkowski and then let the dense distribution of values around it do the tuning a la Bousso-Polchinski (although we do not address this part of the framework here).

Depending on the structure of the induced soft terms in the visible sector, the corresponding matter fields get a zero or non-zero VEV. In turn, the visible sector blow-up mode develops a VEV which can be either vanishing or suppressed by inverse powers of $\mathcal{V}$, justifying the validity of working in the singularity regime.

- The scales determined by this stabilisation procedure acquire realistic values, in the sense that the string scale is close to the standard GUT scale, while the soft terms are either of the order of the $\mathrm{TeV}$-scale (fitting with a sequestered scenario) or of the 
order of the gravitino mass which is heavy (intermediate scale). In both scenarios the lightest volume modulus is heavier $\left(10^{6}-10^{7} \mathrm{GeV}\right)$ than the $\mathrm{TeV}$ scale, e.g. the gaugino mass scale in the sequestered case. Therefore, there is no cosmological moduli problem and the decay of this mode determines reheating dominating late time cosmology.

- In the case with a non-vanishing VEV for a visible sector matter field, its phase becomes a potential candidate for the QCD axion with a decay constant in the allowed range and similar to the (large) value of the gravitino mass $\sim 10^{10} \mathrm{GeV}$. The detailed structure of VEVs for matter fields is more model-dependent, depending in part on the distribution of D3-branes at the $\mathrm{dP}_{6}$ singularity. Since there are many options we will leave this for a future treatment, as well as a discussion of the phenomenology of this class of models.

It is interesting to note that the values for $\left|W_{0}\right|$ found in our analysis are concentrated within the range $1 \lesssim\left|W_{0}\right| \lesssim 100 .{ }^{27}$ This is consistent with the following two important requirements necessary to trust the $4 \mathrm{D}$ low-energy theory. Firstly, we must have $\mathcal{V} \gg\left|W_{0}\right|^{3}$ in order to control the superspace derivative expansion by having a gravitino mass smaller than the Kaluza-Klein scale $M_{\mathrm{KK}}$ [61]. Secondly, we need $\mathcal{V} \gg\left|W_{0}\right|^{6}$ to have a vacuum energy density smaller than $M_{\mathrm{KK}}^{4}[61,84,85]$. However, the last constraint applies only to AdS vacua, and so it does not affect our Minkowski or slightly de Sitter solutions, whereas the first constraint is more generic. For $\left|W_{0}\right| \simeq 100$ it requires a volume of order $\mathcal{V} \gg 10^{6}$. This bound can be easily satisfied in the LVS scenario due to the presence of an exponentially large volume. In the explicit minimisation carried out in section 4.2 , we actually found a minimum with $\mathcal{V} \simeq 10^{8}$.

There are many open questions to be addressed. In order to incorporate cosmological issues into this framework, it would be interesting to considering concrete CY manifolds which allow for a realisation of inflation. Two of the models in appendix B have $h^{1,1}=5$ and are K3-fibred. These are the right features to realise different promising models where the inflaton is a Kähler modulus [86-89]. The moduli space of the open string sector needs to be studied in order to combine moduli stabilisation with a realistic higgsing towards the Standard Model gauge group.

Moreover, in this paper we turned on flux quanta only along three-cycles which are invariant under an appropriate discrete symmetry of the complex structure moduli space. Even in this simpler case, we could find only a few solutions to the minimisation equations which are completely under control, in the sense that the instanton corrections determine only a negligible shift of the VEV of the $u$-moduli. More generically, in order to perform a proper statistical analysis of flux vacua, to understand the distribution of phenomenologically interesting mass scales in the landscape, one should turn on flux quanta on all three-cycles. In addition one needs to be able to find solutions to the complete minimisation equations including also instanton effects. This task is a real technical challenge that we

\footnotetext{
${ }^{27}$ Notice that for technical reasons, we have turned on only a small number of flux quanta. Very small values of $\left|W_{0}\right|$, as required for KKLT, are expected to emerge only when hundreds of flux quanta are switched on.
} 
leave for future work. However, we still think that our paper represents an important step forward in the understanding of crucial questions, like for example the explicit realisation of the Bousso-Polchinski scenario for the cosmological constant [90].

\section{Acknowledgments}

We would like to thank Martin Bies, Mirjam Cvetič, Andres Collinucci, Jim Halverson, Albrecht Klemm, Francesco Muia and Markus Rummel for useful conversations. The research of DK is supported in part by the DOE grant DE-SC0007901 and by Dean's Funds for Faculty Working Group. The work of SK and CM was supported by the DFG under TR33 "The Dark Universe". SK was also supported by the European Union 7th network program Unification in the LHC era (PITN-GA-2009-237920). SK and MC would like to thank ICTP for hospitality.

\section{A Periods and Picard-Fuchs equations}

In this appendix we review some standard facts about mirror symmetry, special geometry and the PF-system that are omitted in the main text for brevity of the discussion, but might prove helpful for the interested reader. We also present explicit formulas for the period vector $\Pi$ and the flux superpotential $W_{\text {flux }}$ in terms of the prepotential $F$. For more details we refer to standard references, e.g. [29, 91, 92]. We closely follow [93].

\section{A.1 Periods as solutions to the Picard-Fuchs equations}

In this section we collect some derivations and explanations that have been skipped in the main text. We will be as brief as possible and refer to the context of the main text frequently, in order to avoid repetitions.

Next we discuss the structure of the complex structure moduli space $\mathcal{M}_{\mathrm{cs}}$ of $M_{3}$. This structure is captured by the dependence of the holomorphic three-form $\Omega$ and its periods $\Pi$ on the complex structure moduli $\underline{z}$. The periods are solutions to the PF differential system. In the following we are mostly interested in the periods at the point of large complex structure $\underline{z} \sim 0$. Invoking mirror symmetry, the periods in the symplectic basis of three-cycles on $M_{3}$ are determined by classical intersections on $\tilde{M}_{3}$. In turn worldsheetinstanton corrections to the Kähler moduli space of $\tilde{M}_{3}$ are predicted by the expansion of the periods of $M_{3}$ at large complex structure/large volume.

The complex structure dependence of the holomorphic three-form $\Omega$ is conveniently parametrized by its periods, introduced in the period expansion (2.4) w.r.t. an integral symplectic basis $\left(\alpha_{K}, \beta^{K}\right)$ of $H^{3}\left(M_{3}, \mathbb{Z}\right)$. The basis elements obey the elementary relations

$$
\int \alpha_{K} \wedge \beta^{L}=-\int \beta^{L} \wedge \alpha_{K}=\delta_{K}^{L}, \quad \int \alpha_{K} \wedge \alpha_{L}=-\int \beta^{L} \wedge \beta^{K}=0 .
$$

In terms of the Poincaré dual basis $\left(A_{K}, B^{L}\right)$ of $H_{3}\left(M_{3}, \mathbb{Z}\right)$ defined via

$$
\int_{A_{K}} \alpha_{L}=-\int_{B^{L}} \beta^{K}=\delta_{L}^{K}
$$


the periods of $\Omega$ can be directly defined by the following integrals

$$
X^{K}=\int_{A_{K}} \Omega, \quad \mathcal{F}_{K}=\int_{B^{K}} \Omega .
$$

The $X^{K}$ form projective coordinates on the complex structure moduli space since $\Omega$ is only defined up to multiplication with $e^{f}$ for a holomorphic function $f$ on the complex structure moduli space. ${ }^{28}$ Mathematically the existence of these coordinates reflects the isomorphism $H^{1}\left(M_{3}, T M_{3}\right) \cong H^{(2,1)}\left(M_{3}\right)$ and the local Torelli theorem. Thus the dimension of complex structure moduli space is $h^{(2,1)}$ and the periods $\underline{\mathcal{F}}$ have to be functions of the $\underline{X}$. Employing Griffiths transversality $[95,96]$ in the form

$$
0=\int_{M_{3}} \Omega \wedge \frac{\partial}{\partial X^{I}} \Omega
$$

it can be shown that $\mathcal{F}_{K}=X^{L} \frac{\partial}{\partial X^{K}} \mathcal{F}_{L}=\frac{\partial}{\partial X^{K}}\left(X^{L} \mathcal{F}_{L}\right)-\mathcal{F}_{I}$, which immediately implies (2.5). The gauge freedom can be fixed by setting $e^{f}=1 / X^{0}$ yielding (2.6) and (2.7).

Next, we note that the natural hermitian metric

$$
h=i \int \Omega \wedge \bar{\Omega}
$$

on the vacuum line bundle $\mathcal{L}$ spanned by $\Omega$ [94] also enters the Kähler potential (2.9) on the complex structure moduli space. Similarly $h$ is used to construct the hermitian/Chern connection on the holomorphic line bundle $\mathcal{L}$,

$$
A_{i}=\partial_{i} \log (h)=-\partial_{i} K_{\mathrm{CS}}=\frac{i}{h}\left(\bar{X}^{K} \partial_{i} \mathcal{F}_{K}-\overline{\mathcal{F}}_{\bar{i}}\right) .
$$

From this one can determine the covariant derivative on $\mathcal{L}$ as $D_{i}=\partial_{i}+\partial_{i} K_{\mathrm{cs}}$, which has to be used e.g. when evaluating the F-terms (2.3). Theses definitions imply that the curvature form of the bundle $\mathcal{L}$ is also the Kähler form on its base manifold, i.e. the complex structure moduli space. This is one central property of $\mathcal{N}=2$ special geometry.

As mentioned in the main text, the main objective in order to control the complex structure sector of a CY three-fold $M_{3}$ is to compute the prepotential (2.6). This is accomplished by solving the associated PF-system, which is constructed as follows. For toric hypersurface the explicit algebraic representation of a family of CY hypersurfaces [97] in a toric variety $\mathbb{P}_{\Delta}$ associated to $\Delta_{4}^{Z}$ reads

$$
P(\underline{x}, \underline{a})=\sum_{p_{j} \in \Delta_{4}^{\tilde{Z}}} a_{j} \prod_{i} x_{i}^{\left\langle v_{i}, p\right\rangle+1} .
$$

Here, we introduced the projective coordinates $x_{i}$ to each vertex $v_{i}$ of $\Delta_{4}^{Z}$ and $p_{j}$ are the integral points in the dual polytope $\Delta_{4}^{\tilde{M}}$. The constants $a_{j}$ are labeled by these points and constitute the complex structure moduli of $M_{3}$. It can be shown, using a residue integral representation for $\Omega[95,96,98-101]$, that directly involve the constraint (A.7), that $\Omega$

\footnotetext{
${ }^{28}$ More precisely $\Omega$ is a section of the vacuum line bundle $\mathcal{L}$ over $\mathcal{M}_{\mathrm{cs}}$, see [94].
} 
obeys the Gelfand-Kapranov-Zelevinski (GKZ) differential system [102, 103]. This system is constituted of the operators $\mathcal{L}_{l}$ and $\mathcal{Z}_{k}$. They are completely determined by the toric data encoded in the points $\tilde{v}_{j}$ and $\ell^{(i)}$-vectors $\ell^{(a)}$ of $\Delta_{4}^{\tilde{M}}$ as

$$
\mathcal{L}_{i}=\prod_{\ell_{j}^{(i)}>0}\left(\frac{\partial}{\partial a_{j}}\right)^{\ell_{j}^{(i)}}-\prod_{\ell_{j}^{(i)}<0}\left(\frac{\partial}{\partial a_{j}}\right)^{-\ell_{j}^{(i)}}, \quad i=1, \ldots, k,
$$

that are the actual PF operators annihilating the periods and operators

$$
\mathcal{Z}_{j}=\sum_{n}\left(\bar{v}_{n}\right)^{j} \vartheta_{n}-\beta_{j}, \quad j=0, \ldots, 4 .
$$

Here $\beta=(-1,0,0,0,0)$ is the so-called exponent of the GKZ-system and $\vartheta_{n}=a_{n} \frac{\partial}{\partial a_{n}}$ denote logarithmic derivatives. We have embedded the points $\tilde{v}_{n}$ into a hypersurface at distance 1 away from the origin by defining $\bar{v}_{n}=\left(1, \tilde{v}_{n}\right)$ so that all zeroth components are $\left(\bar{v}_{n}\right)^{0}=1$.

The differential equations (A.9) are easily solved by introducing coordinates

$$
z^{i}=(-)^{\ell_{0}^{(i)}} \prod_{j=0}^{k+4} a_{j}^{\ell_{j}^{(i)}}, \quad i=1, \ldots k
$$

on the complex structure moduli space of $M_{3}$. In these so-called algebraic coordinates the point of maximally unipotent monodromy, also denoted as the point of large volume/large complex structure, is centred at $z^{i}=0$. Indeed by solving the GKZ-system in these coordinates explicitly using the Frobenius method [104, 105] the obtained solutions have the well-known log-grading. There is a a unique holomorphic power series solution $X^{0}$ and solutions starting with $\left(\ln \left(z^{i}\right)\right)^{k}$ for $k=0, \ldots, 3$. This structure is expected by mirror symmetry due to the shift symmetry of the NS-NS B-field, $b^{i} \mapsto b^{i}+1$, in the Type IIA theory, which implies on the B-model side a maximal logarithmic degeneration of periods near the point $z^{i}=0[104,106]$.

In order to relate these solutions with the geometrical periods $\Pi$ in (2.8) of $\Omega$ in the integral symplectic basis $\left(\alpha_{K}, \beta^{K}\right)$ one employs mirror symmetry. The mirror map (2.14) is constructed by identifying the $X^{i}$ in (2.7) with the single-logarithmic solutions in the $z^{i}$. Then, we have to find the prepotential (2.13) among the solutions of the PF system, which also fixes all other periods by (2.8). It can be argued invoking the matching of D-brane central charges under mirror symmetry [30] that, in a suitable integral basis, the subleading terms of $F$ are given by the classical intersections ${ }^{29}$ (2.15) of the mirror three-fold $\tilde{M}_{3}$, however, with

$$
\mathcal{K}_{i j}=\frac{1}{2} \int_{\tilde{M}_{3}} \iota_{*}\left(c_{1}\left(K_{j}\right)\right) \wedge J_{i} \bmod \mathbb{Z} .
$$

\footnotetext{
${ }^{29}$ We note that the last period in (2.8) only matches the vanishing period at the conifold of the mirror quintic [106] if $\mathcal{K}_{i j}$ is shifted by 8 , which amounts to replace the integrand of $\mathcal{K}_{i j}$ by $\left(J^{2}+c_{2}\left(M_{3}\right)\right)$ with $c_{2}\left(M_{3}\right)$ denoting the second Chern class of $\tilde{M}_{3}[107]$.
} 
Here $c_{1}\left(K_{j}\right)$ denotes the first Chern class of the divisor $K_{j}$ and $\iota_{*}=P_{\tilde{M}_{3}} \circ \iota_{*} \circ P_{K_{j}}^{-1}$ is the Gysin homomorphism where $P_{\tilde{M}_{3}}\left(P_{\tilde{K}_{j}}\right)$ is the Poincaré-duality map on $\tilde{M}_{3}\left(K_{j}\right)$ and $\iota_{*}$ is the push-forward on homology of the embedding $\iota: K_{j} \rightarrow \tilde{M}_{3}$. Thus, $\iota_{*}\left(c_{1}\left(K_{j}\right)\right)$ is a fourform. It is calculated by first noting the projection formula [108] for a map $f: X \rightarrow X^{\prime}$ between two algebraic varieties $X, X^{\prime}$,

$$
f_{*}\left([Y] \cdot f^{*}\left(\left[Y^{\prime}\right]\right)\right)=f_{*}([Y]) \cdot\left[Y^{\prime}\right]
$$

for two subvarieties $Y, Y^{\prime}$ of $X$ respectively $X^{\prime}$ with corresponding homology classes $[Y]$, $\left[Y^{\prime}\right]$. Applying this to the embedding $\iota$ we obtain

$$
\iota_{*}\left(c_{1}\left(K_{j}\right)\right) \cdot J_{i}=\iota_{*}\left(c_{1}\left(K_{j}\right) \cdot \iota^{*}\left(J_{i}\right)\right)=P_{\tilde{M}_{3}}([p t]) \int_{K_{j}} c_{1}\left(K_{j}\right) \iota^{*}\left(J_{i}\right)
$$

where Poincaré duality in the first and second equation is understood. Here we have used that on $K_{j}$ any four-form is proportional to the top form on $K_{j}$ with proportionality factor given by its integral over $K_{j}$. Using the adjunction formula for $K_{j}$ in $\tilde{M}_{3}$ to show $c_{1}\left(K_{j}\right)=-\iota^{*} J_{j}$, we finally obtain

$$
\mathcal{K}_{i j}=-\frac{1}{2} \int_{\tilde{M}_{3}} J_{i} \wedge J_{j}^{2} \bmod \mathbb{Z},
$$

which is precisely the expression in (2.15).

For reference in the main text, we conclude this appendix by presenting the period vector (2.8) in the integral basis that follows from the prepotential (2.13):

$$
\Pi=X^{0}\left(\begin{array}{c}
1 \\
u^{i} \\
\frac{1}{3 !} \mathcal{K}_{i j k} u^{i} u^{j} u^{k}+\mathcal{K}_{i} u^{i}+\mathcal{K}_{0}+\sum_{\beta} n_{\beta}^{0}\left(2 \operatorname{Li}_{3}\left(q^{\beta}\right)-d_{i} u^{i} \operatorname{Li}_{2}\left(q^{\beta}\right)\right) \\
-\frac{1}{2} \mathcal{K}_{i j k} u^{j} u^{k}-\mathcal{K}_{i j} u^{j}+\mathcal{K}_{i}+\sum_{\beta} n_{\beta}^{0} d_{i} \operatorname{Li}_{2}\left(q^{\beta}\right)
\end{array}\right)
$$

We also display flux superpotentials obtained for this period vector

$$
\begin{aligned}
W_{\text {flux }}=X^{0}\left[\hat{N}_{0}+\hat{M}^{0} \mathcal{K}_{0}+\hat{M}^{i} \mathcal{K}_{i}+\left(\hat{N}_{i}-\hat{M}^{j} \mathcal{K}_{i j}+\hat{M}^{0} \mathcal{K}_{i}\right) u^{i}-\frac{1}{2} \hat{M}^{i} \mathcal{K}_{i j k} u^{j} u^{k}\right. \\
\left.\quad+\frac{1}{3 !} \hat{M}^{0} \mathcal{K}_{i j k} u^{i} u^{j} u^{k}+\left(\hat{M}^{i}-\hat{M}^{0} u^{i}\right) \sum_{\beta} d_{i} n_{\beta}^{0} \operatorname{Li}_{2}\left(q^{\beta}\right)+2 \hat{M}^{0} \sum_{\beta} n_{\beta}^{0} \operatorname{Li}_{3}\left(q^{\beta}\right)\right]
\end{aligned}
$$

where the flux $G_{3}=F_{3}-\tau H_{3}$ has been expanded into complex flux quantum numbers $\left(\hat{M}_{K}, \hat{N}^{K}\right)=\left(M_{K}-\tau \tilde{M}_{K}, N^{K}-\tau \tilde{N}^{K}\right)$ formed from the flux quantum numbers $\left(M_{K}, N^{K}\right)$ of $F_{3}$ and $\left(\tilde{M}_{K}, \tilde{N}^{K}\right)$ of $H_{3}$.

\section{B More examples}

In this section we calculate the period vector at large complex structure for selected examples of CY three-folds $X_{3}$ which satisfy the phenomenological criteria established in [16]. These CY three-folds are realized as hypersurfaces in toric varieties. The number of complex structure moduli $\underline{z}$ in the examples ranges between four and five. 


\section{B.1 Example 2}

Here we consider another four moduli example of mirror pairs of CY three-folds $\left(X_{3}, \tilde{X}_{3}\right)$. The polytope $\Delta_{4}^{X}$ and its dual $\Delta_{4}^{\tilde{X}} \mathrm{read}$

$$
\Delta_{4}^{X}=\left(\begin{array}{rrrr|r}
2 & 1 & -1 & -1 & D_{1} \\
2 & 1 & 0 & -2 & D_{2} \\
2 & 2 & -1 & -2 & D_{3} \\
2 & 2 & 0 & -3 & D_{4} \\
0 & 0 & 0 & 1 & D_{5} \\
0 & 0 & 1 & 0 & D_{6} \\
0 & 1 & 0 & 0 & D_{7} \\
-1 & -1 & 0 & 1 & D_{8}
\end{array}\right), \quad \Delta_{4}^{\tilde{X}}=\left(\begin{array}{rrrr|r}
-1 & -1 & -1 & -1 & \tilde{D}_{1} \\
1 & -1 & -1 & -1 & \tilde{D}_{2} \\
1 & -1 & 3 & -1 & \tilde{D}_{3} \\
-3 & 3 & -1 & -1 & \tilde{D}_{4} \\
5 & -1 & -1 & 3 & \tilde{D}_{5} \\
5 & -1 & 3 & 3 & \tilde{D}_{6} \\
1 & 3 & -1 & 3 & \tilde{D}_{7} \\
1 & 3 & 3 & 3 & \tilde{D}_{8}
\end{array}\right) .
$$

Here we have introduced the toric divisors $D_{i}$ respectively $\tilde{D}_{i}$ corresponding to the vertices in $\Delta_{4}^{X}$ respectively $\Delta_{4}^{\tilde{X}}$ in the last column. We note that the polytopes $\Delta_{4}^{X}$ and $\Delta_{4}^{\tilde{X}}$ are congruent. Thus the corresponding toric varieties $\mathbb{P}_{\Delta}$ and $\mathbb{P}_{\tilde{\Delta}}$ differ only by the action of an orbifold group and the mirror construction agrees with the GP orbifold construction of the mirror CY three-fold.

We note again that it is the CY three-fold $X_{3}$ of which we want to calculate the periods on its complex structure moduli space. Its Hodge numbers and Euler number read

$$
h^{(1,1)}=4, \quad h^{(2,1)}=98, \quad \chi\left(X_{3}\right)=-188 .
$$

After demanding invariance under the GP orbifold group, only four of the 98 complex structure moduli remain. The periods left invariant under this orbifold precisely agree with the periods of the mirror $\tilde{X}_{3}$, as explained in section 2.2 .1 , that we calculate next.

All the following geometrical calculations are performed on the Kähler moduli space of $X_{3}$. First we note that there are 16 star-triangulations of $\Delta_{4}^{X}$. By calculating the quartic intersections on the toric ambient variety we see that there are only eight CY phases on $X_{3}$, both with simplicial and non-simplicial Mori cone. We focus in the following on a CY phase with simplicial Mori cone that arises from a single phase of the ambient toric variety. The Mori cone in this phase is generated by

$$
\begin{array}{ll}
\ell^{(1)}=(-1,1,1,-1,0,0,0,0), & \ell^{(2)}=(0,0,0,1,1,0,0,2), \\
\ell^{(3)}=(1,-1,0,0,-1,1,0,0,), & \ell^{(4)}=(1,0,-1,0,-1,0,1,0)
\end{array}
$$

Next, we readily calculate by duality between curves and divisor,

$$
K_{i} \cdot \ell^{(j)}=\delta_{i}^{j},
$$

the generators $K_{i}$ of the Kähler cone. In the chosen triangulation the Kähler cone is spanned by the generators $K_{i}$

$$
K_{1}=D_{1}+D_{2}+D_{3}, \quad K_{2}=D_{1}+D_{2}+D_{3}+D_{4}, \quad K_{3}=D_{1}+D_{3}, \quad K_{4}=D_{1}+D_{2}
$$


in terms of the toric divisors $D_{i}$ introduced in (B.1). In terms of this basis we calculate the classical triple intersections w.r.t. the $(1,1)$-forms $J_{i}$ dual to the $K_{j}$ as

$$
\mathcal{C}_{0}=J_{1}^{2} J_{2}+3 J_{1} J_{2}^{2}+\frac{5 J_{2}^{3}}{3}+2 J_{1} J_{2} J_{3}+2 J_{2}^{2} J_{3}+2 J_{1} J_{2} J_{4}+2 J_{2}^{2} J_{4}+2 J_{2} J_{3} J_{4} .
$$

Analogously we calculate the other classical intersections (2.15) to be

$$
\begin{array}{rlrl}
\mathcal{K}_{12} & =-3, \quad \mathcal{K}_{21}=-1, & \mathcal{K}_{32} & =\mathcal{K}_{42}=-2, \\
\mathcal{K}_{j} J_{j} & =\frac{2}{3} J_{1}+\frac{8}{3} J_{2}+J_{3}+J_{4}, & \mathcal{K}_{02}=-\frac{\zeta(3)}{(2 \pi i)^{3}} 188
\end{array}
$$

with all other $\mathcal{K}_{i j}=0$. We note that these relations hold up to integers as before and the $\mathcal{K}_{i j}$ are symmetric modulo integers.

Finally, the intersections (B.6) and (B.7) allow us to write down the prepotential (2.13) as

$$
\begin{aligned}
F= & -t_{1}^{2} t_{2}-3 t_{1} t_{2}^{2}-\frac{5 t_{2}^{3}}{3}-2 t_{1} t_{2} t_{3}-2 t_{2}^{2} t_{3}-2 t_{1} t_{2} t_{4}-2 t_{2}^{2} t_{4}-2 t_{2} t_{3} t_{4} \\
& +2 t_{1} t_{2}+t_{2} t_{3}+t_{2} t_{4}+\frac{5}{2} t_{2}^{2}+\frac{2}{3} t_{1}+\frac{8}{3} t_{2}+t_{3}+t_{4}-i \zeta(3) \frac{47}{4 \pi^{3}} \\
& +\sum_{\beta} n_{\beta}^{0} \operatorname{Li}_{3}\left(q^{\beta}\right),
\end{aligned}
$$

where $\beta=\left(d_{1}, d_{2}, d_{3}, d_{4}\right)$ in the basis $\beta^{i}$ of effective curves of $H_{2}\left(X_{3}, \mathbf{Z}\right)$ corresponding to the generators (B.3) of the Mori cone and $q^{\beta}=e^{2 \pi i d_{i} t^{i}}$ as before. We also introduced the flat coordinates $t_{i}$ on the Kähler moduli space of $X_{3}$ corresponding to the $\beta^{i}$. The instanton corrections for the Kähler moduli space are determined by solving the PF equations for $\tilde{X}_{3}$ along the lines of section A.1 for the charge vectors (2.21).

\section{B.1.1 The orientifold involution}

The weight matrix for $\Delta_{4}^{X}$, which was given in (B.1), reads as follows:

\begin{tabular}{|c|c|c|c|c|c|c|c|c|}
\hline$z_{1}$ & $z_{2}$ & $z_{3}$ & $z_{4}$ & $z_{5}$ & $z_{6}$ & $z_{7}$ & $z_{8}$ & $D_{\mathrm{eq}_{X}}$ \\
\hline 1 & 0 & 0 & 1 & 0 & 1 & 1 & 4 & 8 \\
\hline 0 & 1 & 0 & 0 & 0 & 0 & 1 & 2 & 4 \\
\hline 0 & 0 & 1 & 0 & 0 & 1 & 0 & 2 & 4 \\
\hline 0 & 0 & 0 & 1 & 1 & 0 & 0 & 2 & 4 \\
\hline
\end{tabular}

Again we note that this phase differs from the one used in (B.3). The Stanley-Reisner ideal in this phase reads:

$$
\text { SR-ideal : } \quad\left\{z_{1} z_{6}, z_{1} z_{7}, z_{2} z_{3}, z_{2} z_{7}, z_{3} z_{6}, z_{4} z_{5} z_{8}\right\}
$$

The hypersurface equation at the symmetric complex structure points is given by:

$$
\begin{aligned}
\mathrm{equ}_{\mathrm{rd}}= & z_{1}^{4} z_{2}^{4} z_{3}^{4} z_{4}^{4}+z_{2}^{4} z_{4}^{4} z_{6}^{4}+z_{1}^{4} z_{2}^{4} z_{5}^{4} z_{6}^{4}+z_{3}^{4} z_{4}^{4} z_{7}^{4}+z_{8}^{2}+\rho_{1} z_{1}^{8} z_{2}^{4} z_{3}^{4} z_{5}^{4} \\
& +\rho_{2} z_{1}^{4} z_{3}^{4} z_{5}^{4} z_{7}^{4}+\rho_{3} z_{5}^{4} z_{6}^{4} z_{7}^{4}+\rho_{4} z_{1} z_{2} z_{3} z_{4} z_{5} z_{6} z_{7} z_{8}+\rho_{4}^{2} z_{1}^{2} z_{2}^{2} z_{3}^{2} z_{4}^{2} z_{5}^{2} z_{6}^{2} z_{7}^{2}
\end{aligned}
$$


The holomorphic involution is given by:

$$
\sigma: \quad\left(z_{2}, z_{6}\right) \leftrightarrow\left(z_{3}, z_{7}\right) .
$$

There is only one $O 7$-plane and it is given by:

$$
\text { O7: } z_{2} z_{6}-z_{3} z_{7}=0,
$$

with $\chi(O 7)=36$. Furthermore, we have $O 3$-planes at

$$
\left\{z_{1}=0, z_{5}=0, z_{2} z_{6}+z_{3} z_{7}=0\right\} \quad \text { and } \quad\left\{z_{1}=0, z_{4}=0, z_{2} z_{6}+z_{3} z_{7}=0\right\}
$$

\section{B.2 Example 3}

In this section we consider a five moduli example of mirror pairs of CY three-folds $\left(X_{3}, \tilde{X}_{3}\right)$. The polytope $\Delta_{4}^{X}$ and its dual $\Delta_{4}^{\tilde{X}} \mathrm{read}$

$$
\Delta_{4}^{X}=\left(\begin{array}{rrrr|r}
3 & 1 & -1 & 1 & D_{1} \\
6 & -1 & 0 & 2 & D_{2} \\
0 & 1 & 0 & 0 & D_{3} \\
6 & -2 & 1 & 2 & D_{4} \\
0 & 0 & 1 & 0 & D_{5} \\
0 & 0 & 0 & 1 & D_{6} \\
-1 & 0 & 0 & -1 & D_{7} \\
3 & 0 & 0 & 1 & D_{8} \\
3 & -1 & 1 & 1 & D_{9}
\end{array}\right), \quad \Delta_{4}^{\tilde{X}}=\left(\begin{array}{rrrr|r}
-1 & -1 & -1 & 2 & \tilde{D}_{1} \\
0 & -1 & -1 & -1 & \tilde{D}_{2} \\
2 & -1 & -1 & -1 & \tilde{D}_{3} \\
2 & -1 & 5 & -1 & \tilde{D}_{4} \\
2 & 5 & -1 & -1 & \tilde{D}_{5} \\
2 & 11 & 11 & -1 & \tilde{D}_{6} \\
2 & 11 & 17 & -1 & \tilde{D}_{7}
\end{array}\right)
$$

Here we have introduced the toric divisors $D_{i}$ respectively $\tilde{D}_{i}$ corresponding to the points in $\Delta_{4}^{X}$ respectively $\Delta_{4}^{\tilde{X}}$ in the last column. We have included two interior points on codimension two and three faces in $\Delta_{4}^{X}$ as the last two points in (B.16). These points are necessary to resolves singularities of the toric ambient space which also descend to the generic CY hypersurface $X_{3}$. We note that the polytopes $\Delta_{4}^{X}$ and $\Delta_{4}^{\tilde{X}}$ are congruent.

The Hodge numbers and Euler number of the CY three-fold $X_{3}$ of which we want to calculate the periods read

$$
h^{(1,1)}=5, \quad h^{(2,1)}=185, \quad \chi\left(X_{3}\right)=-360 .
$$

After demanding invariance under the GP orbifold group, only five of the 185 complex structure moduli remain. The periods left invariant under this orbifold precisely agree with the periods of the mirror $\tilde{X}_{3}$, that we calculate in the next.

All the following calculations are performed on the Kähler moduli space of $X_{3}$. We note that the ambient toric variety $P_{\Delta}$ has 13 inequivalent star-triangulations. As before we calculate the quartic intersections of its toric divisors, noting that all these 13 phases descend to distinct $\mathrm{CY}$ phase of $X_{3}$. We focus here on a particular star-triangulation of $\Delta_{4}^{X}$ with simplicial Mori cone generated by

$$
\begin{array}{ll}
\ell^{(1)}=(0,0,-1,-1,0,0,0,1,1), & \ell^{(2)}=(0,0,0,1,1,0,0,0,-2), \quad \ell^{(3)}=(0,0,1,0,-1,2,3,0,1), \\
\ell^{(4)}=(0,1,1,0,0,0,0,-2,0), & \ell^{(5)}=(1,-1,0,1,0,0,0,-1,0) .
\end{array}
$$


Next, we readily calculate by duality between Mori and Kähler cone, the generators $K_{i}$ of the Kähler cone. In the chosen triangulation the Kähler cone is spanned by the generators $K_{i}$

$$
\begin{array}{ll}
K_{1}=D_{1}-D_{4}+D_{5}+\frac{1}{2} D_{6}, & D_{2}=D_{5}+\frac{1}{2} D_{6}, \\
K_{4}=D_{1}+D_{3}-D_{4}+D_{5}, & K_{5}=D_{1}
\end{array} \quad K_{3}=\frac{1}{2} D_{6}
$$

in terms of the toric divisors $D_{i}$ introduced in (B.1). In terms of this basis we calculate the classical triple intersections as

$$
\begin{aligned}
\mathcal{C}_{0}= & \frac{4 J_{1}^{3}}{3}+4 J_{1}^{2} J_{2}+4 J_{1} J_{2}^{2}+\frac{7 J_{2}^{3}}{6}+4 J_{1}^{2} J_{3}+8 J_{1} J_{2} J_{3}+\frac{7}{2} J_{2}^{2} J_{3}+4 J_{1} J_{3}^{2}+\frac{7}{2} J_{2} J_{3}^{2}+J_{3}^{3} \\
& +\frac{3}{2} J_{1}^{2} J_{4}+3 J_{1} J_{2} J_{4}+\frac{3}{2} J_{2}^{2} J_{4}+3 J_{1} J_{3} J_{4}+3 J_{2} J_{3} J_{4}+\frac{3}{2} J_{3}^{2} J_{4}+\frac{1}{2} J_{1} J_{4}^{2}+\frac{1}{2} J_{2} J_{4}^{2}+\frac{1}{2} J_{3} J_{4}^{2} \\
& +J_{1}^{2} J_{5}+2 J_{1} J_{2} J_{5}+J_{2}^{2} J_{5}+2 J_{1} J_{3} J_{5}+2 J_{2} J_{3} J_{5}+J_{3}^{2} J_{5}+J_{1} J_{4} J_{5}+J_{2} J_{4} J_{5}+J_{3} J_{4} J_{5} .
\end{aligned}
$$

Similarly we calculate the other classical intersections (2.15) to be

$$
\begin{aligned}
\mathcal{K}_{11} & =\mathcal{K}_{12}=\mathcal{K}_{13}=\mathcal{K}_{21}=\mathcal{K}_{31}=-4, & \mathcal{K}_{14} & =\mathcal{K}_{24}=\mathcal{K}_{34}=-\frac{1}{2}, \\
\mathcal{K}_{33} & =-3, & \mathcal{K}_{22}=\mathcal{K}_{23}=\mathcal{K}_{32}=\mathcal{K}_{43}=-\frac{3}{2}, & \mathcal{K}_{51}=\mathcal{K}_{52}=\mathcal{K}_{53}=-1, \\
\mathcal{K}_{j} J_{j} & =\frac{23}{6} J_{1}+\frac{41}{12} J_{2}+3 J_{3}+\frac{3}{2} J_{4}+J_{5}, & \mathcal{K}_{0} & =-\frac{\zeta(3)}{(2 \pi i)^{3}} 360
\end{aligned}
$$

with all other $\mathcal{K}_{i j}=0$. We note that these relations hold up to integers as before and the $\mathcal{K}_{i j}$ are symmetric modulo integers.

Finally, the intersections (B.20) and (B.21) allow us to obtain the prepotential (2.13). For brevity, we do not write out the cubic term by noting that they are identical to (B.20) after replacing $J_{i} \rightarrow-t_{i}$. We obtain

$$
\begin{aligned}
F= & \left.C_{0}\right|_{J_{i}=-t_{i}}+2 t_{1}^{2}+4 t_{1} t_{2}+4 t_{1} t_{3}+\frac{1}{4} t_{1} t_{4}+\frac{1}{4} t_{2} t_{4}+\frac{1}{4} t_{3} t_{4}+\frac{7}{4} t_{2}^{2}+\frac{7}{2} t_{2} t_{3}+\frac{3}{2} t_{3}^{2} \\
& +t_{1} t_{4}+t_{2} t_{4}+t_{3} t_{4}+\frac{1}{2} t_{1} t_{5}+\frac{1}{2} t_{2} t_{5}+\frac{1}{2} t_{3} t_{5}+\frac{23}{6} t_{1}+\frac{41}{12} t_{2}+3 t_{3}+\frac{3}{2} t_{4}+t_{5} \\
& -i \zeta(3) \frac{45}{2 \pi^{3}}+\sum_{\beta} n_{\beta}^{0} \operatorname{Li}_{3}\left(q^{\beta}\right),
\end{aligned}
$$

where $\beta=\left(d_{1}, d_{2}, d_{3}, d_{4}\right)$ in the basis $\beta^{i}$ of effective curves of $H_{2}\left(X_{3}, \mathbf{Z}\right)$ corresponding to the generators (B.18) of the Mori cone and $q^{\beta}=e^{2 \pi i d_{i} t^{i}}$ as before. We also introduced the flat coordinates $t_{i}$ on the Kähler moduli space of $X_{3}$ corresponding to the $\beta^{i}$. The instanton corrections for the Kähler moduli space are determined by solving the PF equations for $\tilde{X}_{3}$ along the lines of section A.1 for the charge vectors (2.21). 


\section{B.2.1 The orientifold involution}

The weight matrix for $\Delta_{4}^{\tilde{X}}$, which was given in (B.16), reads as follows:

\begin{tabular}{|c|c|c|c|c|c|c|c|c|c|}
\hline$z_{1}$ & $z_{2}$ & $z_{3}$ & $z_{4}$ & $z_{5}$ & $z_{6}$ & $z_{7}$ & $z_{8}$ & $z_{9}$ & $D_{\mathrm{eq}_{X}}$ \\
\hline 1 & 1 & 0 & 0 & 1 & 6 & 9 & 0 & 0 & 18 \\
\hline 1 & 0 & 1 & 1 & 0 & 6 & 9 & 0 & 0 & 18 \\
\hline 1 & 0 & 0 & 0 & 0 & 4 & 6 & 0 & 1 & 12 \\
\hline 0 & 1 & 1 & 0 & 0 & 4 & 6 & 0 & 0 & 12 \\
\hline 0 & 0 & 0 & 0 & 0 & 2 & 3 & 1 & 0 & 6 \\
\hline
\end{tabular}

The Stanley-Reisner ideal for the phase of the ambient space, which gives rise to two $\mathrm{dP}_{8}$ divisors and one $\mathrm{dP}_{1}$ divisor on the $\mathrm{CY}$ and respects the K3-fibration structure of the polytope, is given by:

$$
\begin{aligned}
\text { SR-ideal : } \quad & \left\{z_{1} z_{4}, z_{1} z_{5}, z_{1} z_{9}, z_{2} z_{3}, z_{2} z_{5}, z_{4} z_{8}, z_{5} z_{8},\right. \\
& \left.z_{3} z_{4}, z_{4} z_{5}, z_{6} z_{7} z_{8}, z_{2} z_{6} z_{7} z_{9}, z_{3} z_{6} z_{7} z_{9}\right\}
\end{aligned}
$$

The CY hypersurface equation at the symmetric complex structure point becomes:

$$
\begin{aligned}
\mathrm{equ}_{\mathrm{rd}}= & z_{6}^{3}+z_{7}^{2}+z_{1}^{6} z_{2}^{12} z_{4}^{12} z_{8}^{6} z_{9}^{6}+z_{2}^{12} z_{4}^{18} z_{5}^{6} z_{8}^{6} z_{9}^{12}+z_{3}^{12} z_{4}^{6} z_{5}^{18} z_{8}^{6} z_{9}^{12} \\
& +\rho_{1} z_{1}^{6} z_{3}^{12} z_{5}^{12} z_{8}^{6} z_{9}^{6}+\rho_{2} z_{1}^{12} z_{2}^{6} z_{3}^{6} z_{8}^{6}+\rho_{3} z_{2}^{6} z_{3}^{6} z_{4}^{12} z_{5}^{12} z_{8}^{6} z_{9}^{12} \\
& +\rho_{4} z_{1}^{6} z_{2}^{6} z_{3}^{6} z_{4}^{6} z_{5}^{6} z_{8}^{6} z_{9}^{6}+\rho_{5} z_{1}^{4} z_{2}^{4} z_{3}^{4} z_{4}^{4} z_{5}^{4} z_{6} z_{8}^{4} z_{9}^{4}
\end{aligned}
$$

The involution, which is a $\mathbb{Z}_{2}$-symmetry of $\Delta_{4}^{\tilde{X}}$, is given by:

$$
\sigma: \quad\left(z_{2}, z_{4}\right) \leftrightarrow\left(z_{3}, z_{5}\right) .
$$

Under (B.26) the two $\mathrm{dP}_{8}$ divisors $D_{4}$ and $D_{5}$ are mapped onto each other. Furthermore, as a fixed point set we obtain two $O 7$-planes:

$$
O 7_{1}: \quad z_{2} z_{4}-z_{3} z_{5}=0, \quad O 7_{2}: \quad z_{2} z_{4}+z_{3} z_{5}=0
$$

with $\chi\left(O 7_{1}\right)=\chi\left(O 7_{2}\right)=25$ but no $O 3$-planes.

\section{B.3 Example 4}

In this section we consider a three moduli example of mirror pairs of CY three-folds $\left(X_{3}, \tilde{X}_{3}\right)$. The polytope $\Delta_{4}^{X}$ and its dual $\Delta_{4}^{\tilde{X}_{3}} \mathrm{read}$

$$
\Delta_{4}^{X}=\left(\begin{array}{rrrr|r}
6 & 0 & -1 & 2 & D_{1} \\
6 & -1 & 0 & 2 & D_{2} \\
3 & 1 & -1 & 1 & D_{3} \\
3 & -1 & 1 & 1 & D_{4} \\
0 & 0 & 1 & 0 & D_{5} \\
0 & 1 & 0 & 0 & D_{6} \\
0 & 0 & 0 & 1 & D_{7} \\
-1 & 0 & 0 & -1 & D_{8} \\
3 & 0 & 0 & 1 & D_{9}
\end{array}\right), \quad \Delta_{4}^{\tilde{X}}=\left(\begin{array}{rrrr|r}
-1 & -1 & -1 & 2 & \tilde{D}_{1} \\
0 & -1 & -1 & -1 & \tilde{D}_{2} \\
2 & -1 & -1 & -1 & \tilde{D}_{3} \\
2 & -1 & 5 & -1 & \tilde{D}_{4} \\
2 & 5 & -1 & -1 & \tilde{D}_{5} \\
2 & 5 & 11 & -1 & \tilde{D}_{6} \\
2 & 11 & 5 & -1 & \tilde{D}_{7} \\
2 & 11 & 11 & -1 & \tilde{D}_{8}
\end{array}\right)
$$


Here we have introduced the toric divisors $D_{i}$ respectively $\tilde{D}_{i}$ corresponding to the points in $\Delta_{4}^{X}$ respectively $\Delta_{4}^{\tilde{X}}$ in the last column. We note that the last point in $\Delta_{4}^{X}$ is an interior point on a codimension two face, that has resolves a singularity of the toric ambient space which also lies in the generic CY hypersurface $X_{3}$. We note that the polytopes $\Delta_{4}^{X}$ and $\Delta_{4}^{\tilde{X}}$ are congruent. Thus the corresponding toric varieties $\mathbb{P}_{\Delta}$ and $\mathbb{P}_{\tilde{\Delta}}$ differ only by the action of an orbifold group and as before the mirror construction agrees with the GP orbifold construction of the mirror CY three-fold.

We recall again that it is the CY three-fold $X_{3}$ of which we need to calculate the periods on its complex structure moduli space. Its Hodge numbers and Euler number read

$$
h^{(1,1)}=5, \quad h^{(2,1)}=185, \quad \chi\left(X_{3}\right)=-360 .
$$

After demanding invariance under the GP orbifold group, only five of the 185 complex structure moduli remain. The periods left invariant under this orbifold precisely agree with the periods of the mirror $\tilde{X}_{3}$, that we calculate in the next.

All the following topological calculations are performed on the Kähler moduli space of $X_{3}$. First we note that there are 18 star-triangulation of $\Delta_{4}^{X}$ yielding $18 \mathrm{CY}$ phase on $X_{3}$. We chose one phase with Mori cone generated by

$$
\begin{array}{ll}
\ell^{(1)}=(-1,1,1,0,0,0,2,3,0), & \ell^{(2)}=(0,0,0,1,-1,1,0,0,-1), \\
\ell^{(3)}=(0,0,1,0,1,-1,0,0,-1), & \ell^{(4)}=(0,1,0,-1,1,0,0,0,-1), \\
\ell^{(5)}=(1,-1,-1,0,0,0,0,0,1) . &
\end{array}
$$

Next, we readily calculate by duality between curves and divisor, the generators $K_{i}$ of the Kähler cone. In the chosen triangulation the Kähler cone is spanned by the generators

$$
\begin{array}{ll}
K_{1}=2 D_{1}+2 D_{2}+D_{3}+D_{4}+D_{9}, & K_{2}=D_{1}+D_{2}+D_{4}, \quad K_{3}=D_{1}+D_{3}, \\
K_{4}=D_{1}+D_{2}, & K_{5}=3 D_{1}+2 D_{2}+D_{3}+D_{4}+D_{9}
\end{array}
$$

in terms of the toric divisors $D_{i}$ introduced in (B.28). In terms of this basis we calculate the classical triple intersections as

$$
\begin{aligned}
\mathcal{C}_{0}= & J_{1}^{3}+\frac{3}{2} J_{1}^{2} J_{2}+\frac{1}{2} J_{1} J_{2}^{2}+J_{1}^{2} J_{3}+J_{1} J_{2} J_{3}+J_{1}^{2} J_{4}+J_{1} J_{2} J_{4}+J_{1} J_{3} J_{4}+\frac{7}{2} J_{1}^{2} J_{5}+3 J_{1} J_{2} J_{5} \\
& +\frac{1}{2} J_{2}^{2} J_{5}+2 J_{1} J_{3} J_{5}+J_{2} J_{3} J_{5}+2 J_{1} J_{4} J_{5}+J_{2} J_{4} J_{5}+J_{3} J_{4} J_{5}+\frac{7}{2} J_{1} J_{5}^{2}+\frac{3}{2} J_{2} J_{5}^{2}+J_{3} J_{5}^{2} \\
& +J_{4} J_{5}^{2}+\frac{7 J_{5}^{3}}{6} .
\end{aligned}
$$


Analogously we calculate the other classical intersections (2.15) to be

$$
\begin{aligned}
\mathcal{K}_{11} & =-3 \\
\mathcal{K}_{12} & =\mathcal{K}_{52}=-\frac{1}{2} \\
\mathcal{K}_{21} & =\mathcal{K}_{25}=-\frac{3}{2} \\
\mathcal{K}_{31} & =\mathcal{K}_{35}=\mathcal{K}_{41}=\mathcal{K}_{45}=-1, \\
\mathcal{K}_{51} & =\mathcal{K}_{55}=\mathcal{K}_{15}=-\frac{7}{2}, \\
\mathcal{K}_{j} J_{j} & =3 J_{1}+\frac{3}{2} J_{2}+J_{3}+J_{4}+\frac{41}{12} J_{5}, \\
\mathcal{K}_{0} & =-\frac{\zeta(3)}{(2 \pi i)^{3}} 360
\end{aligned}
$$

with all other $\mathcal{K}_{i j}=0$. We note that these relations hold up to integers as before.

Finally, the intersections (B.32) and (B.33) allow us to obtain the prepotential (2.13). The cubic terms are obtained by replacing $J_{i} \rightarrow-t_{i}$ in (B.32) and we obtain

$$
\begin{aligned}
F= & \left.C_{0}\right|_{J_{i}=-t_{i}}+\frac{3}{2} t_{1}^{2}+t_{1} t_{2}+\frac{1}{2} t_{1} t_{3}+\frac{1}{2} t_{1} t_{4}+\frac{7}{2} t_{1} t_{5}+t_{2} t_{5}+\frac{1}{2} t_{3} t_{5}+\frac{7}{4} t_{5}^{2} \\
& +3 t_{1}+\frac{3}{2} t_{2}+t_{3}+t_{4}+\frac{41}{12} t_{5}-i \zeta(3) \frac{45}{2 \pi^{3}}+\sum_{\beta} n_{\beta}^{0} \operatorname{Li}_{3}\left(q^{\beta}\right),
\end{aligned}
$$

where $\beta=\left(d_{1}, d_{2}, d_{3}, d_{4}\right)$ in the basis $\beta^{i}$ of effective curves of $H_{2}\left(X_{3}, \mathbf{Z}\right)$ corresponding to the generators (B.30) of the Mori cone and $q^{\beta}=e^{2 \pi i d_{i} t^{i}}$ as before. We also introduced the flat coordinates $t_{i}$ on the Kähler moduli space of $X_{3}$ corresponding to the $\beta^{i}$. The instanton corrections for the Kähler moduli space are determined by solving the PF equations for $\tilde{X}_{3}$ along the lines of section A.1 for the charge vectors (2.21).

\section{B.3.1 The orientifold involution}

The weight matrix for $\Delta_{4}^{\tilde{X}}$, which was given in (B.28), reads as follows:

\begin{tabular}{|c|c|c|c|c|c|c|c|c|c|}
\hline$z_{1}$ & $z_{2}$ & $z_{3}$ & $z_{4}$ & $z_{5}$ & $z_{6}$ & $z_{7}$ & $z_{8}$ & $z_{9}$ & $D_{\mathrm{eq}_{X}}$ \\
\hline 1 & 0 & 0 & 0 & 1 & 0 & 4 & 6 & 0 & 12 \\
\hline 0 & 1 & 1 & 0 & 1 & 0 & 6 & 9 & 0 & 18 \\
\hline 0 & 1 & 0 & 0 & 0 & 1 & 4 & 6 & 0 & 12 \\
\hline 0 & 0 & 1 & 1 & 0 & 0 & 4 & 6 & 0 & 12 \\
\hline 0 & 0 & 0 & 0 & 0 & 0 & 2 & 3 & 1 & 6 \\
\hline
\end{tabular}

The Stanley-Reisner ideal for the phase of the ambient space, which gives rise to two $\mathrm{dP}_{8}$ divisors and one $\mathrm{dP}_{1}$ divisor on the $\mathrm{CY}$ and respects the K3-fibration structure of the polytope, is given by:

SR-ideal : $\quad\left\{z_{1} z_{5}, z_{1} z_{6}, z_{2} z_{3}, z_{2} z_{5}, z_{2} z_{6}, z_{2} z_{9}, z_{3} z_{4}\right.$, $\left.z_{3} z_{5}, z_{5} z_{9}, z_{7} z_{8} z_{9}, z_{1} z_{4} z_{7} z_{8}, z_{4} z_{6} z_{7} z_{8}\right\}$ 
The CY hypersurface equation at the symmetric complex structure point reads as follows:

$$
\begin{aligned}
\mathrm{equ}_{\mathrm{rd}}= & z_{7}^{3}+z_{8}^{2}+z_{2}^{6} z_{4}^{12} z_{5}^{12} z_{6}^{6} z_{9}^{6}+z_{1}^{6} z_{3}^{12} z_{5}^{6} z_{6}^{12} z_{9}^{6}+z_{3}^{6} z_{4}^{6} z_{5}^{12} z_{6}^{12} z_{9}^{6} \\
& +\rho_{1} z_{1}^{12} z_{2}^{6} z_{3}^{12} z_{6}^{6} z_{9}^{6}+\rho_{2} z_{1}^{6} z_{2}^{12} z_{4}^{12} z_{5}^{6} z_{9}^{6}+\rho_{3} z_{1}^{12} z_{2}^{12} z_{3}^{6} z_{4}^{6} z_{9}^{6} \\
& +\rho_{4} z_{1}^{6} z_{2}^{6} z_{3}^{6} z_{4}^{6} z_{5}^{6} z_{6}^{6} z_{9}^{6}+z_{1}^{4} z_{2}^{4} z_{3}^{4} z_{4}^{4} z_{5}^{4} z_{6}^{4} z_{7} z_{9}^{4}
\end{aligned}
$$

The involution which leaves the ambient variety invariant and maps the two $\mathrm{dP}_{8}$ 's at $D_{2}$ and $D_{5}$ onto each other is given by:

$$
\sigma: \quad\left(z_{1}, z_{2}\right) \leftrightarrow\left(z_{6}, z_{5}\right)
$$

The fixed point set of this involution is given by one $O 7$-plane:

$$
\text { O7: } \quad z_{1} z_{2}-z_{6} z_{5}=0
$$

with $\chi(O 7)=24$ and $2 \times 1$ plus $2 \times 3 O 3$-planes at

$$
\begin{array}{ll}
\left\{z_{4}=z_{1} z_{2}+z_{5} z_{6}=z_{9}=0\right\}, & \left\{z_{3}=z_{1} z_{2}+z_{5} z_{6}=z_{9}=0\right\}, \\
\left\{z_{4}=z_{1} z_{2}+z_{5} z_{6}=z_{8}=0\right\}, & \left\{z_{3}=z_{1} z_{2}+z_{5} z_{6}=z_{8}=0\right\} .
\end{array}
$$

Open Access. This article is distributed under the terms of the Creative Commons Attribution License (CC-BY 4.0), which permits any use, distribution and reproduction in any medium, provided the original author(s) and source are credited.

\section{References}

[1] G. Aldazabal, L.E. Ibáñez, F. Quevedo and A.M. Uranga, D-branes at singularities: a bottom up approach to the string embedding of the standard model, JHEP 08 (2000) 002 [hep-th/0005067] [INSPIRE].

[2] L.E. Ibanez and A.M. Uranga, String theory and particle physics: an introduction to string phenomenology, Cambridge University Press, Cambridge U.K. (2012) [INSPIRE].

[3] S.B. Giddings, S. Kachru and J. Polchinski, Hierarchies from fluxes in string compactifications, Phys. Rev. D 66 (2002) 106006 [hep-th/0105097] [INSPIRE].

[4] S. Kachru, R. Kallosh, A.D. Linde and S.P. Trivedi, de Sitter vacua in string theory, Phys. Rev. D 68 (2003) 046005 [hep-th/0301240] [INSPIRE].

[5] V. Balasubramanian, P. Berglund, J.P. Conlon and F. Quevedo, Systematics of moduli stabilisation in Calabi-Yau flux compactifications, JHEP 03 (2005) 007 [hep-th/0502058] [INSPIRE].

[6] A. Maharana and E. Palti, Models of particle physics from type IIB string theory and F-theory: a review, Int. J. Mod. Phys. A 28 (2013) 1330005 [arXiv:1212.0555] [InSPIRE].

[7] J.P. Conlon, A. Maharana and F. Quevedo, Towards realistic string vacua, JHEP 05 (2009) 109 [arXiv:0810.5660] [INSPIRE].

[8] S. Krippendorf, M.J. Dolan, A. Maharana and F. Quevedo, D-branes at toric singularities: model building, Yukawa couplings and flavour physics, JHEP 06 (2010) 092 [arXiv: 1002.1790] [INSPIRE]. 
[9] C.P. Burgess, S. Krippendorf, A. Maharana and F. Quevedo, Radiative fermion masses in local D-brane models, JHEP 05 (2011) 103 [arXiv: 1102.1973] [INSPIRE].

[10] M.J. Dolan, S. Krippendorf and F. Quevedo, Towards a systematic construction of realistic D-brane models on a del Pezzo singularity, JHEP 10 (2011) 024 [arXiv:1106.6039] [INSPIRE].

[11] T. Weigand, Lectures on F-theory compactifications and model building, Class. Quant. Grav. 27 (2010) 214004 [arXiv: 1009.3497] [INSPIRE].

[12] J.J. Heckman, Particle physics implications of F-theory, Ann. Rev. Nucl. Part. Sci. 60 (2010) 237 [arXiv:1001.0577] [InSPIRE].

[13] C. Vafa, Geometry of grand unification, arXiv:0911.3008 [INSPIRE].

[14] M. Wijnholt, F-theory, GUTs and chiral matter, arXiv:0809.3878 [INSPIRE].

[15] M. Cicoli, C. Mayrhofer and R. Valandro, Moduli stabilisation for chiral global models, JHEP 02 (2012) 062 [arXiv:1110.3333] [INSPIRE].

[16] M. Cicoli, S. Krippendorf, C. Mayrhofer, F. Quevedo and R. Valandro, D-branes at del Pezzo singularities: global embedding and moduli stabilisation, JHEP 09 (2012) 019 [arXiv:1206.5237] [INSPIRE].

[17] M. Cicoli, S. Krippendorf, C. Mayrhofer, F. Quevedo and R. Valandro, D3/D7 branes at singularities: constraints from global embedding and moduli stabilisation, JHEP 07 (2013) 150 [arXiv:1304.0022] [INSPIRE].

[18] M. Cicoli, S. Krippendorf, C. Mayrhofer, F. Quevedo and R. Valandro, The web of D-branes at singularities in compact Calabi-Yau manifolds, JHEP 05 (2013) 114 [arXiv:1304.2771] [INSPIRE].

[19] C. Mayrhofer, Compactifications of type IIB string theory and F-theory models by means of toric geometry, Ph.D. Thesis, Technische Universität Wien (2010).

[20] M. Cicoli, M. Kreuzer and C. Mayrhofer, Toric K3-fibred Calabi-Yau manifolds with del Pezzo divisors for string compactifications, JHEP 02 (2012) 002 [arXiv:1107.0383] [INSPIRE].

[21] X. Gao and P. Shukla, On classifying the divisor involutions in Calabi-Yau threefolds, JHEP 11 (2013) 170 [arXiv:1307.1139] [INSPIRE].

[22] A. Giryavets, S. Kachru, P.K. Tripathy and S.P. Trivedi, Flux compactifications on Calabi-Yau threefolds, JHEP 04 (2004) 003 [hep-th/0312104] [INSPIRE].

[23] F. Denef, M.R. Douglas and B. Florea, Building a better racetrack, JHEP 06 (2004) 034 [hep-th/0404257] [INSPIRE].

[24] B.R. Greene and M.R. Plesser, Duality in Calabi-Yau moduli space, Nucl. Phys. B 338 (1990) 15 [inSPIRE].

[25] F. Denef and M.R. Douglas, Distributions of flux vacua, JHEP 05 (2004) 072 [hep-th/0404116] [INSPIRE].

[26] J. Louis, M. Rummel, R. Valandro and A. Westphal, Building an explicit de Sitter, JHEP 10 (2012) 163 [arXiv:1208.3208] [INSPIRE].

[27] D. Martinez-Pedrera, D. Mehta, M. Rummel and A. Westphal, Finding all flux vacua in an explicit example, JHEP 06 (2013) 110 [arXiv: 1212.4530] [INSPIRE]. 
[28] G. Curio, A. Klemm, D. Lüst and S. Theisen, On the vacuum structure of type-II string compactifications on Calabi-Yau spaces with H fluxes, Nucl. Phys. B 609 (2001) 3 [hep-th/0012213] [INSPIRE].

[29] A. Klemm, Topological string theory on Calabi-Yau threefolds, PoS (RTN2005) 002 [INSPIRE].

[30] P. Mayr, Phases of supersymmetric D-branes on Kähler manifolds and the McKay correspondence, JHEP 01 (2001) 018 [hep-th/0010223] [INSPIRE].

[31] T.W. Grimm, T.-W. Ha, A. Klemm and D. Klevers, Computing brane and flux superpotentials in F-theory compactifications, JHEP 04 (2010) 015 [arXiv:0909.2025] [INSPIRE].

[32] A. Klemm, Instanton.

[33] M. Kreuzer and H. Skarke, PALP: a package for analyzing lattice polytopes with applications to toric geometry, Comput. Phys. Commun. 157 (2004) 87 [math. NA/0204356] [INSPIRE].

[34] A.P. Braun, J. Knapp, E. Scheidegger, H. Skarke and N.-O. Walliser, PALP - a user manual, arXiv:1205.4147 [INSPIRE].

[35] V. Braun and A.Y. Novoseltsev, Toric varieties framework for Sage, The Sage Development Team (2012), http://www.sagemath.org/doc/reference/schemes/sage/schemes/toric/variety.html.

[36] P. Berglund, S.H. Katz and A. Klemm, Mirror symmetry and the moduli space for generic hypersurfaces in toric varieties, Nucl. Phys. B 456 (1995) 153 [hep-th/9506091] [InSPIRE].

[37] P. Shanahan, The Atiyah-Singer index theorem, Lect. Notes Math. 638 (1978) 1.

[38] R. Blumenhagen, A. Collinucci and B. Jurke, On instanton effects in F-theory, JHEP 08 (2010) 079 [arXiv: 1002.1894] [INSPIRE].

[39] J. Distler and B.R. Greene, Aspects of $(2,0)$ string compactifications, Nucl. Phys. B 304 (1988) 1 [inSPIRE].

[40] I. Brunner and K. Hori, Orientifolds and mirror symmetry, JHEP 11 (2004) 005 [hep-th/0303135] [INSPIRE].

[41] V. Balasubramanian, P. Berglund, V. Braun and I. Garcia-Etxebarria, Global embeddings for branes at toric singularities, JHEP 10 (2012) 132 [arXiv:1201.5379] [INSPIRE].

[42] M. Wijnholt, Large volume perspective on branes at singularities, Adv. Theor. Math. Phys. 7 (2004) 1117 [hep-th/0212021] [INSPIRE].

[43] D.R. Gulotta, Properly ordered dimers, R-charges and an efficient inverse algorithm, JHEP 10 (2008) 014 [arXiv:0807.3012] [INSPIRE].

[44] A. Hanany and D. Vegh, Quivers, tilings, branes and rhombi, JHEP 10 (2007) 029 [hep-th/0511063] [INSPIRE].

[45] M. Yamazaki, Brane tilings and their applications, Fortschr. Phys. 56 (2008) 555 [arXiv:0803.4474] [INSPIRE].

[46] R. Blumenhagen, M. Cvetič, S. Kachru and T. Weigand, D-brane instantons in type II orientifolds, Ann. Rev. Nucl. Part. Sci. 59 (2009) 269 [arXiv:0902.3251] [InSPIRE].

[47] D.S. Freed and E. Witten, Anomalies in string theory with D-branes, Asian J. Math. 3 (1999) 819 [hep-th/9907189] [INSPIRE].

[48] P. Berglund and I. Garcia-Etxebarria, D-brane instantons on non-spin cycles, JHEP 01 (2013) 056 [arXiv:1210.1221] [inSPIRE]. 
[49] A. Collinucci, M. Kreuzer, C. Mayrhofer and N.-O. Walliser, Four-modulus 'Swiss cheese' chiral models, JHEP 07 (2009) 074 [arXiv:0811.4599] [INSPIRE].

[50] M. Bianchi, A. Collinucci and L. Martucci, Magnetized E3-brane instantons in F-theory, JHEP 12 (2011) 045 [arXiv:1107.3732] [InSPIRE].

[51] M. Bianchi, G. Inverso and L. Martucci, Brane instantons and fluxes in F-theory, JHEP 07 (2013) 037 [arXiv:1212.0024] [INSPIRE].

[52] H. Jockers and J. Louis, D-terms and F-terms from D7-brane fluxes, Nucl. Phys. B 718 (2005) 203 [hep-th/0502059] [INSPIRE].

[53] R. Blumenhagen, B. Körs, D. Lüst and S. Stieberger, Four-dimensional string compactifications with D-branes, orientifolds and fluxes, Phys. Rept. 445 (2007) 1 [hep-th/0610327] [INSPIRE].

[54] R. Blumenhagen, M. Cvetič and T. Weigand, Spacetime instanton corrections in $4 D$ string vacua: the seesaw mechanism for D-brane models, Nucl. Phys. B 771 (2007) 113 [hep-th/0609191] [INSPIRE].

[55] L.E. Ibáñez and A.M. Uranga, Neutrino Majorana masses from string theory instanton effects, JHEP 03 (2007) 052 [hep-th/0609213] [INSPIRE].

[56] B. Florea, S. Kachru, J. McGreevy and N. Saulina, Stringy instantons and quiver gauge theories, JHEP 05 (2007) 024 [hep-th/0610003] [INSPIRE].

[57] R. Blumenhagen, S. Moster and E. Plauschinn, Moduli stabilisation versus chirality for MSSM like type IIB orientifolds, JHEP 01 (2008) 058 [arXiv:0711.3389] [INSPIRE].

[58] R. Blumenhagen, V. Braun, T.W. Grimm and T. Weigand, GUTs in type IIB orientifold compactifications, Nucl. Phys. B $\mathbf{8 1 5}$ (2009) 1 [arXiv:0811.2936] [InSPIRE].

[59] D.J. Bates, D. Brake and M. Niemerg, Paramotopy: parallel parameter homotopy via Bertini, available at http://www.paramotopy.com.

[60] D.J. Bates, J.D. Hauenstein, A.J. Sommese and C.W. Wampler, Bertini: software for numerical algebraic geometry, available at http://dx.doi.org/10.7274/r0h41pb5.

[61] M. Cicoli, J.P. Conlon, A. Maharana and F. Quevedo, A note on the magnitude of the flux superpotential, JHEP 01 (2014) 027 [arXiv: 1310.6694] [INSPIRE].

[62] T.W. Grimm and J. Louis, The effective action of $N=1$ Calabi-Yau orientifolds, Nucl. Phys. B 699 (2004) 387 [hep-th/0403067] [INSPIRE].

[63] M. Graña, T.W. Grimm, H. Jockers and J. Louis, Soft supersymmetry breaking in Calabi-Yau orientifolds with D-branes and fluxes, Nucl. Phys. B 690 (2004) 21 [hep-th/0312232] [INSPIRE].

[64] N. Arkani-Hamed, M. Dine and S.P. Martin, Dynamical supersymmetry breaking in models with a Green-Schwarz mechanism, Phys. Lett. B 431 (1998) 329 [hep-ph/9803432] [INSPIRE].

[65] R. Blumenhagen, J.P. Conlon, S. Krippendorf, S. Moster and F. Quevedo, SUSY breaking in local string/F-theory models, JHEP 09 (2009) 007 [arXiv: 0906.3297] [INSPIRE].

[66] K. Becker, M. Becker, M. Haack and J. Louis, Supersymmetry breaking and $\alpha^{\prime}$-corrections to flux induced potentials, JHEP 06 (2002) 060 [hep-th/0204254] [INSPIRE].

[67] M. Berg, M. Haack and B. Körs, String loop corrections to Kähler potentials in orientifolds, JHEP 11 (2005) 030 [hep-th/0508043] [INSPIRE]. 
[68] G. von Gersdorff and A. Hebecker, Kähler corrections for the volume modulus of flux compactifications, Phys. Lett. B 624 (2005) 270 [hep-th/0507131] [INSPIRE].

[69] L. Anguelova, C. Quigley and S. Sethi, The leading quantum corrections to stringy Kähler potentials, JHEP 10 (2010) 065 [arXiv:1007.4793] [INSPIRE].

[70] T.W. Grimm, D. Klevers and M. Poretschkin, Fluxes and warping for gauge couplings in F-theory, JHEP 01 (2013) 023 [arXiv: 1202.0285] [INSPIRE].

[71] I. Garcia-Etxebarria, H. Hayashi, R. Savelli and G. Shiu, On quantum corrected Kähler potentials in F-theory, JHEP 03 (2013) 005 [arXiv: 1212.4831] [INSPIRE].

[72] T.W. Grimm, R. Savelli and M. Weissenbacher, On $\alpha^{\prime}$ corrections in $N=1$ F-theory compactifications, Phys. Lett. B $\mathbf{7 2 5}$ (2013) 431 [arXiv: 1303.3317] [INSPIRE].

[73] J. Halverson, H. Jockers, J.M. Lapan and D.R. Morrison, Perturbative corrections to Kähler moduli spaces, arXiv:1308.2157 [INSPIRE].

[74] M. Berg, M. Haack and E. Pajer, Jumping through loops: on soft terms from large volume compactifications, JHEP 09 (2007) 031 [arXiv: 0704.0737] [INSPIRE].

[75] M. Cicoli, J.P. Conlon and F. Quevedo, Systematics of string loop corrections in type IIB Calabi-Yau flux compactifications, JHEP 01 (2008) 052 [arXiv:0708.1873] [INSPIRE].

[76] J.P. Conlon and F. Quevedo, Gaugino and scalar masses in the landscape, JHEP 06 (2006) 029 [hep-th/0605141] [INSPIRE].

[77] J.P. Conlon, S.S. Abdussalam, F. Quevedo and K. Suruliz, Soft SUSY breaking terms for chiral matter in IIB string compactifications, JHEP 01 (2007) 032 [hep-th/0610129] [INSPIRE].

[78] D. Baumann et al., On D3-brane potentials in compactifications with fluxes and wrapped D-branes, JHEP 11 (2006) 031 [hep-th/0607050] [INSPIRE].

[79] G.D. Coughlan, W. Fischler, E.W. Kolb, S. Raby and G.G. Ross, Cosmological problems for the Polonyi potential, Phys. Lett. B 131 (1983) 59 [INSPIRE].

[80] T. Banks, D.B. Kaplan and A.E. Nelson, Cosmological implications of dynamical supersymmetry breaking, Phys. Rev. D 49 (1994) 779 [hep-ph/9308292] [INSPIRE].

[81] B. de Carlos, J.A. Casas, F. Quevedo and E. Roulet, Model independent properties and cosmological implications of the dilaton and moduli sectors of $4 D$ strings,

Phys. Lett. B 318 (1993) 447 [hep-ph/9308325] [INSPIRE].

[82] M. Cicoli, J.P. Conlon and F. Quevedo, Dark radiation in LARGE volume models, Phys. Rev. D 87 (2013) 043520 [arXiv: 1208.3562] [INSPIRE].

[83] T. Higaki and F. Takahashi, Dark radiation and dark matter in large volume compactifications, JHEP 11 (2012) 125 [arXiv:1208.3563] [INSPIRE].

[84] J.P. Conlon, F. Quevedo and K. Suruliz, Large-volume flux compactifications: moduli spectrum and D3/D7 soft supersymmetry breaking, JHEP 08 (2005) 007 [hep-th/0505076] [INSPIRE].

[85] S.P. de Alwis, Constraints on LVS compactifications of IIB string theory, JHEP 05 (2012) 026 [arXiv: 1202.1546] [INSPIRE].

[86] J.P. Conlon and F. Quevedo, Kähler moduli inflation, JHEP 01 (2006) 146 [hep-th/0509012] [INSPIRE]. 
[87] J.R. Bond, L. Kofman, S. Prokushkin and P.M. Vaudrevange, Roulette inflation with Kähler moduli and their axions, Phys. Rev. D 75 (2007) 123511 [hep-th/0612197] [InSPIRE].

[88] M. Cicoli, C.P. Burgess and F. Quevedo, Fibre inflation: observable gravity waves from IIB string compactifications, JCAP 03 (2009) 013 [arXiv:0808.0691] [INSPIRE].

[89] M. Cicoli, F.G. Pedro and G. Tasinato, Poly-instanton inflation, JCAP 12 (2011) 022 [arXiv:1110.6182] [INSPIRE].

[90] R. Bousso and J. Polchinski, Quantization of four form fluxes and dynamical neutralization of the cosmological constant, JHEP 06 (2000) 006 [hep-th/0004134] [INSPIRE].

[91] M. Mariño, Chern-Simons theory and topological strings, Rev. Mod. Phys. 77 (2005) 675 [hep-th/0406005] [INSPIRE].

[92] M. Vonk, A mini-course on topological strings, hep-th/0504147 [INSPIRE].

[93] D. Klevers, Holomorphic couplings in non-perturbative string compactifications, Fortschr. Phys. 60 (2012) 3 [arXiv:1106.6259] [INSPIRE].

[94] K. Hori et al., Mirror symmetry, Clay Mathematics Monographs, AMS, Providence U.S.A. (2003) [INSPIRE].

[95] P.A. Griffiths, On the periods of certain rational integrals: I, Annals Math. 90 (1969) 460.

[96] P.A. Griffiths, On the periods of certain rational integrals: II, Annals Math. 90 (1969) 496.

[97] V.V. Batyrev, Dual polyhedra and mirror symmetry for Calabi-Yau hypersurfaces in toric varieties, J. Alg. Geom. 3 (1994) 493 [alg-geom/9310003] [INSPIRE].

[98] P.A. Griffiths, Periods of integrals on algebraic manifolds, I, Am. J. Math. 90 (1968) 568.

[99] P.A. Griffiths, Periods of integrals on algebraic manifolds, II, Am. J. Math. 90 (1968) 805.

[100] P.A. Griffiths, Periods of integrals on algebraic manifolds, III, Publ. Math. 38 (1970) 125.

[101] P.A. Griffiths, Periods of integrals on algebraic manifolds: summary of main results and discussion of open problems, B. Am. Math. Soc. 76 (1970) 228.

[102] I. Gelfand, M. Kapranov and A. Zelevinsky, Hypergeometric functions and toric varieties, Funct. Anal. Appl. 23 (1989) 524.

[103] I. Gelfand, M. Kapranov and A. Zelevinsky, Generalized Euler integrals and A-hypergeometric functions, Adv. Math. 84 (1990) 255.

[104] S. Hosono, A. Klemm, S. Theisen and S.-T. Yau, Mirror symmetry, mirror map and applications to Calabi-Yau hypersurfaces, Commun. Math. Phys. 167 (1995) 301 [hep-th/9308122] [INSPIRE].

[105] S. Hosono, A. Klemm, S. Theisen and S.-T. Yau, Mirror symmetry, mirror map and applications to complete intersection Calabi-Yau spaces, Nucl. Phys. B 433 (1995) 501 [hep-th/9406055] [INSPIRE].

[106] P. Candelas, X.C. De La Ossa, P.S. Green and L. Parkes, A pair of Calabi-Yau manifolds as an exactly soluble superconformal theory, Nucl. Phys. B 359 (1991) 21 [INSPIRE].

[107] M.-x. Huang, A. Klemm and S. Quackenbush, Topological string theory on compact Calabi-Yau: modularity and boundary conditions, Lect. Notes Phys. 757 (2009) 45 [hep-th/0612125] [INSPIRE].

[108] W. Fulton, Intersection theory, Springer, Berlin Germany (1998). 